\title{
Prediction of High Speed Propeller Flow Fields Using a Three-Dimensional
} Euler Analysis

Lawrence J. Bober

Lewis Research Center

Cleveland, Ohio

and

Denny S. Chaussee and Paul Kutler

Ames Research Center

Moffett Field, California

\section{FOR REFRENCE}

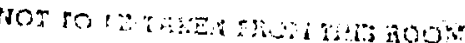

Prepared for the

Twenty-First Aerospace Sciences Conference sponsored by the American Institute of Aeronautics and Astronautics

Reno, Nevada, January 10-13, 1983

\section{LBRARY BOPY}

.

LANGLEY RESEAFCH CENTER

LIBRARY, NASA

HAMPTON, VIRGINIA

\section{N/Sก}


PREDICTION OF HIGH SPEED PROPELLER FLOW FIELDS

USING A THREE-DIMENSIONAL EULER ANALYSIS

\author{
by Lawrence J. Bober * \\ National Aeronautics and Space Administration \\ Lewis Research Center \\ Cleveland, Ohio 44135

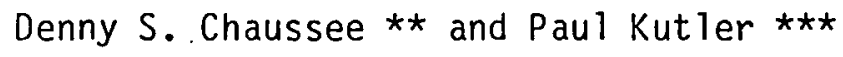 \\ National Aeronautics and Space Administration \\ Ames Research Center \\ Moffett Field, California
}

\begin{abstract}
To overcome the limitations of classical propeller theory, a computer program, NASPROP-E, has been developed which solves for the flow field surrounding a multi-bladed propeller and axisymmetric nacelle combination using a in finite-difference method. The governing equations are the three-dimensional unsteady Euler equations written in a cylindrical coordinate system. They are marched in time until a steady state solution is obtained. The Euler equations require no special treatment to model the blade work vorticity. The equations are solved using an implicit approximate factorization method.

Numerical results are presented which have greatly increased the understanding of high speed propeller flow fields. Numerical results for swirl angle downstream of the propeller and propeller power coefficient are higher than experimental results. The radial variation of swirl angle, however, is in reasonable agreement with the experimental results. The predicted variation of power coefficient with blade angle agrees very well with data.
\end{abstract}

ऋ Head, PropelTer Research Section; member AIAA.

$\star \star$ Research Scientist; member AIAA.

$\star \star \star$ Chief, Applied Computational Aerodynamics Branch; Associate Fellow, AIAA. 
NOMENCLATURE

A

b

B

C

C

$\mathrm{CN}$

D

e

E

G

G

$\left.{ }_{j}^{H_{r_{z}}}, i_{r}, i_{\phi}\right)$

$k_{j}, i=0,3$

LEA

$n$

$p$

$\mathrm{q}_{\infty}$

$q_{j}, i=1,5$

$\mathrm{Q}$

$\mathrm{R}$

$(t, z, r, \phi)$

u

U

v

V

$\vec{V}$

$\nabla$

w

$W$

B $3 / 4$

$\gamma$

$\Delta \beta$

$\Delta \mathrm{p}$

$\Delta \xi, \Delta \eta, \Delta \zeta$

$\rho$

$\sigma_{i}, i=1,5$

$(\tau, \xi, n, \zeta)$

$\varepsilon_{\mathrm{i}}$

$\omega$

Subscripts

i

j

$\infty$

Jacobian matrix, $\partial E / \partial Q$

blade width or chord

Jacobian matrix, $\partial F / \partial Q$

speed of sound, $(\gamma p / \rho)^{1 / 2}$

Jacobian matrix, $\partial G / \partial Q$

Courant number (see Eq. (7))

diameter of propeller

total energy per unit volume

vector of flux quantities in $\xi$-direction

vector of flux quantities in $n$-direction

vector of flux quantities in $\zeta$-direction

vector of source terms created by cylindrical

generalized coordinate transformation

rothalpy

unit normal vector of cylindrical coordinate system Jacobian or advance ratio, $\left(U_{\infty} / n D\right)$

generalized matrices

leading edge alignment

rotational speed, revolutions per second

static pressure

dynamic pressure, $0.5 \rho_{\infty} v_{\infty}^{2}$

components of $Q$

vector dependent variable of integration

blade tip radius

independent variables, cylindrical coordinates

physical velocity in z-direction

contravariant velocity defined in Eq. (2)

physical velocity in or $r$-direction

contravariant velocity defined in Eq. (2)

velocity vector, $u i_{z}+v i_{r}+w i_{\phi}$

vector form of the contravariant velocity

physical velocity in or $\phi$-direction

contravariant velocity defined in Eq. (2)

blade angle at $r / R=0.75$

ratio of specific heats

blade twist

incremental pressure

computational mesh spacing

density

eigenvalues of gas-dynamic equations (see Eq. (6))

transformed independent variables (Eq. (1))

explicit smoothing coefficient in implicit algorithm

implicit smoothing coefficient in implicit algorithm angular velocity

integer mesh point location in 5 -direction

integer mesh point location in $n$-direction

integer mesh point location in $\zeta$-direction

free stream conditions 


\section{INTRODUCTION}

The continuing quest for improved propulsive efficiency for subsonic aircraft has caused a growing interest in the propeller as an alternative to the turbofan engine at cruise Mach numbers up to 0.8 . Numerous mission studies have shown that advanced turboprop powered aircraft offer a potential 15 to 25 percent trip fuel savings over comparable technology high bypass ratio turbo$f$ an powered aircraft at Mach 0.8. Aerodynamic design of propellers for high subsonic flight speeds results in a large number of highly swept blades and a nacelle and spinner designed to have favorable interference effects on the propeller.

Interest from a computational point of view was stimulated by the need for understanding the transonic flow phenomena around propellers and the potential for a more efficient computer generated design. over the years, computational procedures for solving fluid flow problems provided an inexpensive but accurate means of determining the aerodynamic characteristics of complex configurations. In addition, they have provided the designer with an effective tool for maximizing aerodynamic efficiency without the expense of actually building and testing numerous designs. Finally, computational methods have often been capable of providing information not readily obtainable from experiments.

The advanced high speed propeller model is a good example of the type of configuration for which it is difficult to experimentally obtain aerodynamic information needed for performance analyses and design. The blades are virtually impossible to adequately instrument because of the high rotational velocities, and their relatively small thickness. Also, details of the surrounding flow field can only be obtained by performing costly and time consuming flow field surveys. Computationally, however, the entire flow field can be determined from a single solution of the governing equations including near and far-field effects which can be used in acoustic analysis programs and blade surface pressure distributions which can be used in structural and aerodynamic design analysis programs.

The theoretical development and subsequent numerical solutions described herein are concerned with simulating the inviscid flow about a wind tunnel model which consisted of an eight-bladed propeller and spinner with an axisymmetric nacelle (instead of the conventional three-dimensional nacelle with inlet). Such a configuration thus requires that only the flow about a single blade be computed because of periodicity.

\section{DERIVATION OF GOVERNING EQUATIONS}

To enhance numerical accuracy and efficiency, a nonorthogonal coordinate transformation of the governing equations in a particular base coordinate system is employed. This maps the surface of the nacelle and both sides of the blade onto constant coordinate surfaces which facilitates the application of boundary conditions and permits grid point clustering at the body where gradients of the dependent variables are expected to undergo rapid changes. Use of such transformations, in addition, permits utilization of uniform discretization formulas and well-ordered interior grid point solution algorithms. Under this transformation the equations can still be written in conservation-law form to take advantage of the shock capturing properties. 
The basic orthogonal coordinate system utilized is cylindrical with $z$ oriented along the rotational axis, $r$ extending radially outward from the $z$-axis, and $\$$ the meridional angle measured from a vertical plane (see fig. 1). It should be reiterated that for this study, only the flow between two of the blades is computed, i.e., between the pressure side of one blade and the suction side of the next blade, because of periodicity. The cylindrical coordinates are transformed to align the blade and nacelle surfaces with various computational planes according to the following:

$$
\begin{aligned}
& \tau=t \\
& \xi=\xi(t, z, r, \phi) \\
& \eta=n(t, z, r, \phi) \\
& \zeta=\zeta(t, z, r, \phi)
\end{aligned}
$$

This generalized nonorthogonal coordinate transformation maps the spinner and nacelle onto a constant $n-p l a n e$ and each side of the blade, i.e., the suction and pressure sides, onto parts of a constant $\zeta-p l a n e$. The remaining parts of the constant $\zeta$-planes are periodic surfaces. The radial far-stream and outflow boundaries are situated far enough from the prop-fan to minimize the reflection of waves.

The governing partial differential equations in weak conservation-law form for cylindrical coordinates under the assumptions of inviscid compressible flow and a perfect, non-heat conducting gas for the transformation given in Eq. (1) are:

$$
\begin{aligned}
& Q_{T}+E_{\xi}+F_{\eta}+G_{\zeta}+H=0 \\
& Q=\frac{1}{J}\left[\begin{array}{c}
\rho \\
\rho u \\
\rho v \\
\rho w \\
e
\end{array}\right], \quad E=\frac{1}{J}\left[\begin{array}{c}
\rho U \\
\rho u U+p \xi_{z} \\
\rho v U+p \xi_{r} \\
\rho w U+p \xi_{\phi} / r \\
(e+p) U-p \xi_{t}
\end{array}\right] \\
& F=\frac{1}{J}\left[\begin{array}{c}
\rho V \\
\rho u V+p n_{z} \\
\rho u V+p n_{r} \\
\rho W V+p n_{\phi} / r \\
\left(e^{+} p\right) V-p n_{t}
\end{array}\right], G=\frac{1}{J}\left[\begin{array}{c}
\rho W \\
\rho W+p \zeta_{z} \\
\rho v W+p \zeta_{r} \\
\rho w W+p \zeta_{\phi} / r \\
(e+p) W-p \zeta_{t}
\end{array}\right], H=\frac{1}{J r}\left[\begin{array}{c}
\rho v \\
\rho u v \\
\rho\left(v^{2}-w^{2}\right) \\
2 \rho v w \\
(e+p) v
\end{array}\right]
\end{aligned}
$$


and

$$
\begin{aligned}
& U=\xi_{t}+u \xi_{z}+v \xi_{r}+w \xi_{\phi} / r \\
& v=n_{t}+u n_{z}+v \eta_{r}+w m_{\phi} / r \\
& W=\zeta_{t}+u \zeta_{z}+v \zeta_{r}+w \zeta_{\phi} / r
\end{aligned}
$$

$U, V$, and $W$ are the contravariant velocities written without metric normalization. $J$ is the transformation Jacobian and is defined below. Use of the Euler equations in conservation laws form guarantees the accurate calculation of the shock waves occurring in the flow field.

In the conservative variables of Eq. $(2(a))$, the pressure $p$ is nondimen sionalized by $p_{\infty}$, the density $\rho$ by $\rho_{\infty}$, and the cylindrical velocity components $u, v$, and $w$ by $a_{\infty} / \sqrt{\gamma}$ where $a_{\infty}$ is the free-stream speed of sound $\left(a_{\infty}^{2}=\gamma p_{\infty} / \rho_{\infty}\right)$ and $\gamma$ is the ratio of specific heats. 0ther quantities made dimensionless are the time $t$ by $a_{\infty} /(D \sqrt{\gamma})$ and the angular velocity $\omega$ by $D \sqrt{\gamma} / a_{\infty}$. The pressure, density, and velocity components are related to the total energy per unit volume e by the following equation for an ideal gas:

$$
e=p /(r-1)+\rho\left(u^{2}+v^{2}+w^{2}\right) / 2
$$

The metrics of Eq. (2(b)) are obtained by the chain rule expansion of $z_{\xi}, r_{\xi}$, etc. and solved for $\xi_{z},{ }^{\xi}$, etc. to yield the following expressions:

$$
\begin{aligned}
& \xi_{t}=-z_{\tau} \xi_{z}-r_{\tau} \xi_{r}-\phi_{\tau} \xi_{\phi} \quad \xi_{z}=\left(r_{\eta} \phi_{\zeta}-\phi_{\eta} r_{\zeta}\right) / I \\
& n_{t}=-z_{\tau}{ }^{n} z-r_{\tau}{ }^{n} r-\phi_{\tau} n_{\phi} \quad \xi_{r}=\left(\phi_{n} z_{\zeta}-z_{n} \phi_{\zeta}\right) / I \\
& \zeta_{t}=-z_{\tau}{ }^{\zeta}-r_{\tau}{ } r-\phi_{\tau}{ }_{\phi} \quad \xi_{\phi}=\left(z_{n} r_{\zeta}-z_{\zeta}{ }_{\eta}\right) / I \\
& n_{z}=\left(\phi_{\xi} r_{\zeta}-r_{\xi} \phi_{\zeta}\right) / I \quad \zeta_{z}=\left(r_{\xi} \phi_{\eta}-\phi_{\xi} r_{\eta}\right) / I \\
& { }_{r}=\left(z_{\xi} \phi_{\zeta}-\phi_{\xi} z_{\zeta}\right) / I \quad{ }_{r}=\left(z_{\eta} \phi_{\xi}-\phi_{\eta} z_{\xi}\right) / I \\
& n_{\phi}=\left(r_{\xi} z_{\zeta}-z_{\xi} r_{\zeta}\right) / I \quad \zeta_{\phi}=\left(z_{\xi} r_{\eta}-r_{\xi} z_{\eta}\right) / I
\end{aligned}
$$


where

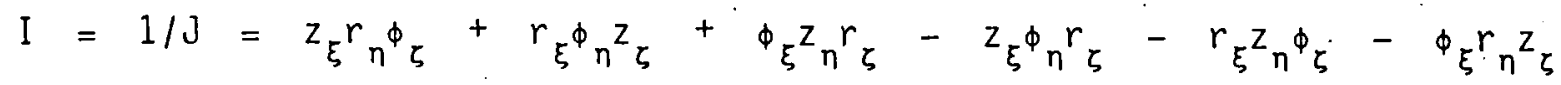

The quantity $\phi_{\tau}$ is the rotational or angular velocity of the propeller.

In general, the metrics of Eq. (4) are not known analytically and must be determined numerically at each step of the integration procedure. To accomplish this, second order central-difference formulas are used at interior points and three-point one-sided formulas are used at the boundaries.

The implicit algorithm to be discussed below requires the analytic determination of the Jacobians $A=\partial E / \partial Q, B=\partial F / \partial Q$, and $C=\partial G / \partial Q$. These Jacobian matrices result from the time linearization of $E, F$, and $G$ in deriving the numerical algorithm. The Jacobian matrices can be written in general as follows:

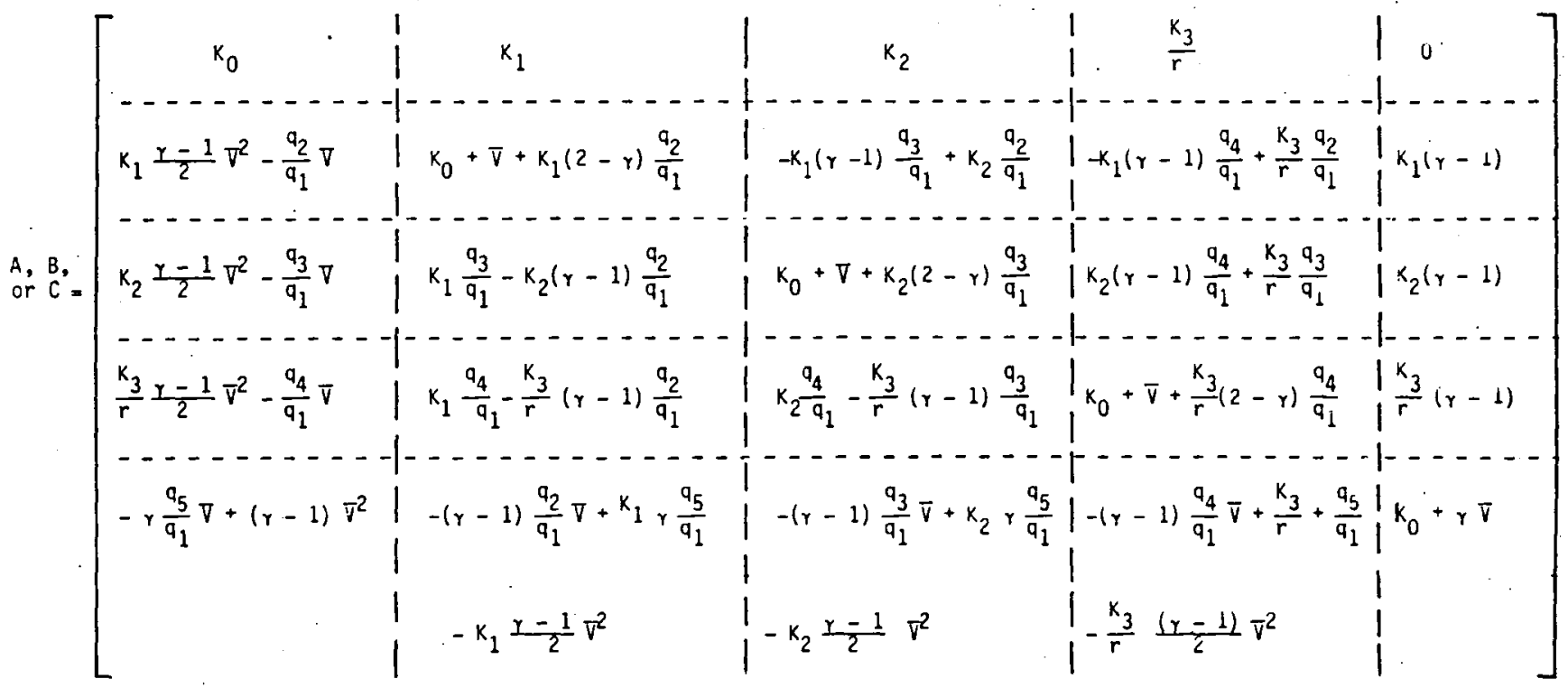

where $\bar{V}=\left(k_{1} q_{2}+k_{2} q_{3}+\frac{k_{3}}{r} q_{4}\right) / q_{1}$ and $\bar{v}^{2}=\left(q_{2}^{2}+q_{3}^{2}+q_{4}^{2}\right) / q_{1}^{2}$.

The terms $q_{i}, i=1,5$ are the components of the dependent variable of integration $Q$ in Eq. (2). To obtain, for example, $A$, let $k_{0}=\xi_{t}, k_{1}=$ $\xi_{z}, \quad k_{2}=\xi_{r}$ and $k_{3}=\xi_{\phi}$ in Eq. (5). 
As a measure of the productivity of the implicit algorithm to be described below compared to that of conventional explicit procedures, the value of the Courant number $c$ an be computed. It requires the eigenvalues of the matrices $A, B$, and $C$. They are as follows:

$$
\sigma_{1,2,3}=k_{0}+u k_{1}+v k_{2}+\frac{w}{r} k_{3}
$$

and

$$
\sigma_{4,5}=k_{0}+u k_{1}+v k_{2}+\frac{w}{r} k_{3} \pm c\left|k_{1}^{2}+k_{2}^{2}+\left(\frac{k_{3}}{r}\right)^{2}\right| 1 / 2
$$

where for $A, k_{0}=\xi_{t}$, etc.; for $B, k_{0}=n_{t}$, etc.; for $C, k_{0}=\xi_{t}$, etc.; and $c$ equals the local speed of sound $\left(c^{2}=r p / p\right)$.

The Courant number is defined as follows:

$$
C N=\Delta \tau /\left|\sigma_{\max }\right|
$$

where it is assumed that $\Delta \xi=\Delta \eta=\Delta \zeta=1$ and $\sigma_{\max }$ is the maximum of the eigenvalues of all the nodal points.

For the present problem, Eq. $(2(a))$ is solved in a time-asymptotic fashion with interest only in the steady-state solution. This is a result of the mesh rotating with the blade and the fact that a cylindrical coordinate system is used. The $Q_{\tau}$ term of Eq. (2(a)) approaches zero as $\tau$ becomes large, thus establishing a convergence criterion.

\section{NUMERICAL METHOD}

The numerical algorithm used to solve the conservation-law form of the Euler equations is based on a class of completely implicit, noniterative, ADI (alternating direction implicit) schemes developed by Lindemuth and Killeen (ref. 1), Briley and McDonald (refs. 2 and 3), and Beam and Warming. (refs. 4 and 5). The particular method is a generalization of a conservative, approximate factorization scheme in the "delta" form (ref. 4). The procedure has been successfully applied by Steger (ref. 8), Pulliam and Steger (ref. 9), and numerous others (refs. 10 and 11) for viscous flows. Use of the implicit procedure helps remove the stiffness of the problem introduced by a fine mesh. Thus, for this problem the implicit procedure permits an integration stepsize large enough to obtain steady-state solutions in considerably fewer iterations than conventional explicit procedures.

As applied to Eq. (2) the implicit, spatially factored algorithm using Euler implicit time differencing takes the form 


$$
\begin{aligned}
& \left|I+\Delta t \delta_{\xi} A^{n}-\varepsilon_{i}\left(J^{-1} \nabla_{\xi} \Delta_{\xi} J\right)^{n}\right|\left|I+\Delta t \delta_{n} B^{n}-\varepsilon_{i}\left(J^{-1} \nabla_{n} \Delta_{n} J\right)\right| \\
& \left|I+\Delta t \delta_{\zeta} C^{n}-\varepsilon_{j}\left(J^{-1} \nabla_{\zeta} \Delta_{\zeta} J\right)^{n}\right|\left(Q^{n+1}-Q^{n}\right)= \\
& -\Delta t\left|\delta_{\zeta} E^{n}+\delta_{\eta} F^{n}+H^{n}+\delta_{\zeta} G^{n}\right|-\frac{\varepsilon}{8}\left(J^{n}\right)^{-1}\left|\left(\nabla_{\xi} \Delta_{\xi}\right)^{2}+\left(\nabla_{\zeta} \Delta_{\zeta}\right)^{2}+\left(\nabla_{n} \Delta_{n}\right)^{2}\right|(J Q)^{n}
\end{aligned}
$$

where $A, B$, and $C$ are the Jacobian matrices $\partial E / \partial U$, $\partial F / \partial U$, and $\partial G / \partial U$, respectively, and $I$ is the identity matrix. $\delta_{\xi}$ and $\delta_{\eta}$ are second-order centraldifference operators, and $\Delta$ and $\nabla$ represent the conventional forward and backward difference operators. The quantities multiplied by $\varepsilon_{i}$ on the left-hand side of the equation represent implicit second-order smoothing terms (refs. 8 and 9 ) while the quantity multipled by $\varepsilon_{e}$ on the right-hand side represents an explicit fourth-order smoothing term.

The solution of Eq. (8) consists of first forming the right-hand term (also called the steady-state or explicit part) at each grid point. Each of the implicit operators, (for instance $\left\{I+\Delta t \delta_{\xi} A^{n}-\varepsilon_{i}\left(J^{-1} \nabla_{\xi} \Delta_{\xi} J\right)^{n_{j}}\right)$ represent blocktridiagonal matrices which must be inverted sequentially to obtain $\Delta Q^{n}=Q^{n+1}-Q^{n}$. A block lower-upper-decomposition algorithm, see Isaacson and Keller (ref. 12), is used for the inversion process.

For steady-state calculations $\Delta Q^{n}=Q^{n+1}-Q^{n}+0$ and the solution then satisfies the steady-state finite-difference equations (right-hand side of Eq. (8)). Linear stability analysis shows unconditional stability for the "delta" form of the implicit approximate factorization algorithm. In actual practice, though, time step limitations are encountered, although they are usually much less stringent than explicit stability bounds. The smoothing terms have been added to control nonlinear instabilities. Choices of the time step $\Delta t$ and the smoothing coefficients $\left(\varepsilon_{e}, \varepsilon_{j}\right)$ are usually dictated by experience. Linear analys is for the smoothing terms does show that for $\varepsilon_{j}=0, \varepsilon_{e}$ is bounded $\left(\varepsilon_{e}<1 / 4\right)$ for stability, but for $\varepsilon_{j} \gg \varepsilon_{e}, \varepsilon_{e}$ is not constrained to any limit for stability.

The metric terms are obtained using second-order finite-differences for terms such as, $z_{\xi}$, in Eqs. (4). Fourth-order accuracy in the steady-state $c a n$ be obtained $\xi_{n}$ an efficient manner by introducing fourth-order finite differences for the convective terms on the right-hand side of Eq. (8), while retaining second-order differences on the left-hand side. For Euler implicit time differencing this is a stable and accurate process. For more details on the above, see Pulliam and Steger (ref. 9). 
The major portion of the computational work in an implicit finite difference algorithm is contained in the solution of the set of simultaneous equations, (i.e., the block tridiagonal equations). When even an implicit algorithm is applied to a system of partial differential equations, one obtains block matrix-vector equations that are complicated and time-consuming to solve. A method for uncoupling the solution process through a diagonalization of the block-matrix structure has been presented by Pulliam and Chaussee (ref. 11). The method which is employed here was originally applied to an implicit approximate-factorization algorithm for the twoand threedimensional inviscid Euler equations in general curvilinear coordinates. Details on the theory can be found in reference 13 .

Pulliam and Chaussee (ref. 11) show that the diagonal algorithm retains the stability and convergence properties of the standard algorithm. Also, since the right-hand side is identical for the two algorithms, the steadystate solution is not affected by the diagonalization. Reductions in CPU time of up to 30 percent can be realized by using the diagonal form.

\section{GEOMETRY AND MESH GENERATION DESCRIPTIONS}

The generation of a computational mesh for calculating the flow through and around a propeller configuration consists of three steps. Step one is composed of generating an $x-y-z$ system of points given the ccoordinates of the nacelle and blades. Step two consists of using the $x-y-z$ locations of step one and to form a system of parametric cubic patches. Step three takes the patches of step two and, based on the specified clustering and/or stretching of points, creates a mesh which is used by the computational code.

The geometry for the blade, spinner, and nacelle must be specified. The geometry is described by the blade twist in degrees, $\Delta B$, relative to the blade angle at 75 percent blade radius, the local blade chord ratio, b/D, and the leading edge alignment ratio, LEA/D, where $D$ is the tip diameter. The thickness ratio distribution for each spanwise section is also required.

The uitimate goal of the mesh generation scheme is to smoothly transition from the blade with its twist and taper to a set of surfaces which have : 1) zero twist or taper above an $r / D=1,2$ ) a slope $d \phi / d z=0$ at the stagnation point of the nacelle, and 3) zero slope at some specified distance downstream from the blade trailing edge. The two transition curves which are employed to accomplish this are parabolic and cubic in nature. These curves allow for end points and/or slopes at the end points to be specified in the transitioning process.

Once these surfaces have been specified, it is an easy matter to extend the surfaces at constant values of any specified outer boundary. Usually this boundary is chosen to be far enough removed from the configuration such that there are no outer interactions affecting the computation.

This whole procedure is automated with only outer boundary points and/or slopes specified in addition to the coordinates of the blade and nacelle surfaces. 
Step 2 is a self sufficient part of the grid generation program. An $x-y-z$ set of points from step one is all that is necessary to create a series of parametric cubic patches which describes the entire mesh system in terms of cubic equations.

In the third and final step, the computational mesh used in the flow field code is created from the series of patches from step 2.

Details of the mesh generation procedures are given in reference 13 .

\section{TANGENCY CONDITIONS}

It is necessary to satisfy the tangency condition at both the blade and nacelle surfaces. In the case of the blade surface, this requires the contravariant velocity component $W$ to be zero (no flow through the surface). To obtain the cylindrical velocity components velocity components along the $(x, y, z, t)=$ constant surface, the following system of equations must be solved:

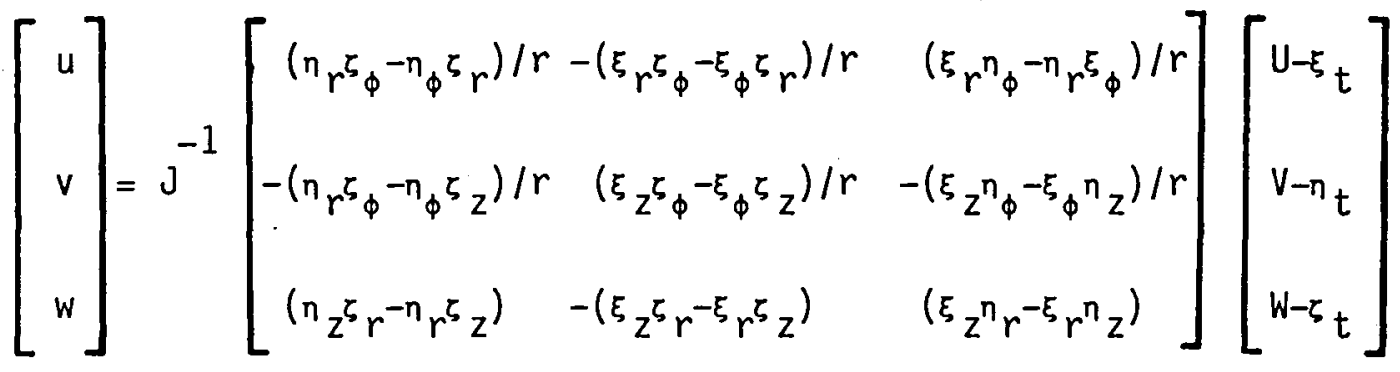

To solve these equations, a complete set of contravariant velocities, $U$, $v$, and $w$, must be known. The velocities $u, v$ and $w$ at the surface are determined by linear extrapolation from known data in the interier. The values of $U$ and $V$ are then determined from equations $(2(c))$ and values of $u, v$ and $w$ are calculated from Eqs. (9) with $W$ set to zero.

Flow velocities at the blade tip and trailing edge are determined in the same manner as the rest of the blade. No Kutta condition is applied at these locations since the velocity of the fluid relative to the blades can be supersonic. Imposition of periodicity at the mesh point just off the blade effectively applies the Kutta condition at that location.

Due to insufficient mesh points to resolve the rapid change from low velocity to very high velocity in the leading edge region of the blade, flow properties at the leading edge of the blade are determined by averaging the values immediately upstream and downstream of the leading edge.

At the nacelle surface, the contravariant velocity $V$ is required to be zero to satisfy the tangency condition. In the same manner as for the blade surfaces, $U$ and $W$ are determined on the surface and $u, v$ and $w$ are determined from Eq. (2(c)) with $V$ set to zero. At the blade nacelle juncture, both $V$ and $W$ are set to zero before determining the surface velocities.

Values of pressure and density on the blade and nacelle surfaces (except for the blade leading edge) are determined by linear extrapolation from values in the interior of the computational region. 
As a check on the accuracy of this procedure the fluid total rothalpy is monitored throughout the flow field. The total rothalpy ${ }_{r}$ is defined as the total enthalpy in the rotating frame of reference or:

$$
H_{r}=\frac{\gamma}{\gamma-1} \frac{p}{\rho}+\frac{1}{2}\left(u^{2}+v^{2}+w^{2}-2 r \omega w\right)
$$

In a flow with uniform free stream, the total rothalpy is constant throughout the flow field.

\section{SUBSONIC OUTFLOW}

The relationships enforcing boundary conditions at the subsonic outflow are determined from the governing partial differential equations using the method of characteristics. The equations, in non-conservation-law form cast in cylindrical coordinates for nonviscous, non-heat conducting, adiabatic, compressible flow, under the transformation of $\mathrm{Eq}$. (1) are:

Continuity

$$
\begin{gathered}
\frac{\partial p}{\partial \tau}+U \frac{\partial p}{\partial \xi}+V \frac{\partial p}{\partial \eta}+W \frac{\partial p}{\partial \zeta}+\rho C^{2}\left[\frac{\partial u}{\partial \xi} \frac{\partial \xi}{M z}+\frac{\partial V}{\partial \xi} \frac{\partial \xi}{M r}+\frac{1}{r} \frac{\partial W}{\partial \xi} \frac{\partial \xi}{\partial \phi}+\frac{\partial u}{\partial \eta} \frac{\partial \eta}{\partial z}\right. \\
\left.+\frac{\partial V}{\partial \eta} \frac{\partial \eta}{\partial r}+\frac{1}{r} \frac{\partial W}{\partial \eta} \frac{\partial \eta}{\partial \phi}+\frac{\partial u}{\partial \zeta} \frac{\partial \zeta}{\partial z}+\frac{\partial V}{\partial \zeta} \frac{\partial \zeta}{\partial r}+\frac{1}{r} \frac{\partial W}{\partial \zeta} \frac{\partial \zeta}{\partial \phi}\right]=0
\end{gathered}
$$

\section{$\underline{\xi}$ - Momentum}

$\frac{\partial u}{\partial \tau}+U \frac{\partial u}{\partial \xi}+V \frac{\partial u}{\partial \eta}+W \frac{\partial u}{\partial \zeta}+\frac{1}{\rho}\left[\frac{\partial p}{\partial \xi} \frac{\partial \xi}{\partial z}+\frac{\partial p}{\partial \eta} \frac{\partial \eta}{\partial z}+\frac{\partial p}{\partial \zeta} \frac{\partial \zeta}{\partial z}\right]=0$

n- Momentum

$$
\frac{\partial V}{\partial \tau}+U \frac{\partial V}{\partial \xi}+V \frac{\partial V}{\partial \eta}+W \frac{\partial V}{\partial \zeta}+\frac{1}{\rho}\left[\frac{\partial p}{\partial \xi} \frac{\partial \xi}{\partial r}+\frac{\partial p}{\partial \eta} \frac{\partial \eta}{\partial r}+\frac{\partial p}{\partial \zeta} \frac{\partial \zeta}{\partial r}\right]=0
$$

\section{¿-Momentum}

$\frac{\partial W}{\partial \tau}+U \frac{\partial W}{\partial \xi}+V \frac{\partial W}{\partial \eta}+W \frac{\partial W}{\partial \zeta}+\frac{1}{\rho} \frac{1}{r}\left[\frac{\partial p}{\partial \xi} \frac{\partial \xi}{\partial \phi}+\frac{\partial p}{\partial \eta} \frac{\partial \eta}{\partial \phi}+\frac{\partial p}{\partial \zeta} \frac{\partial \zeta}{\partial \phi}\right]=0$

where the contravariant velocities are defined in Eq. (2(c)). The details for deriving the characteristics and the compatibility equations from Eqs. (11 to 14 ) can be found in reference 13 . 
For the outflow (exit) boundary conditions, which is a finite distance downstream of the blade trailing edge, a constant pressure is specified. The U-C characteristic brings information into the control volume from outside when $U<c$. Thus one dependent variable such as the back pressure must be specified. The remaining dependent variables are determined by using the up running characteristic $(+)$ in the compatibility relationship to solve for the axial velocity, $u$. The momentum equations are used to solve for the remaining velocity components, $v$ and $w$, and the energy equation is solved for the density.

The uprunning characteristic form of the compatibility equation, with the appropriate $\lambda$ and $\mu_{i}$ 's is written as

$$
p_{\tau}+\left[U+c \sqrt{\xi_{z}^{2}+\xi_{r}^{2}+\frac{\xi_{\phi}}{r}}\right] p_{\xi}
$$$$
+\frac{\rho c \xi_{z}}{\sqrt{\xi_{z}^{2}+\xi_{r}^{2}+\left(\frac{\xi_{\phi}}{r}\right)^{2}}}\left\{u_{\tau}+\left[u+c \sqrt{\xi_{z}^{2}+\xi_{r}^{2}+\left(\frac{\xi_{\phi}}{r}\right)^{2}}\right] u_{\xi}\right\}
$$$$
+\frac{\rho c \xi r}{\sqrt{\xi_{z}^{2}+\xi_{r}^{2}+\left(\frac{\xi_{\phi}}{r}\right)^{2}}}\left\{v_{\tau}+\left[u+c \sqrt{\xi_{z}^{2}+\xi_{r}^{2}+\left(\frac{\xi_{\phi}}{r}\right)^{2}}\right] v_{\xi}\right\}
$$$$
+\frac{\rho \xi_{\phi}}{r \sqrt{\xi_{z}^{2}+\xi_{r}^{2}+\frac{\xi_{\phi}}{r}}}\left\{w_{\tau}+\left[u+c \sqrt{\xi_{z}^{2}+\xi_{r}^{2}+\left(\frac{\xi_{\phi}}{r}\right)^{2}}\right] w_{\xi}\right\}
$$$$
=\frac{\rho c}{\sqrt{\xi_{z}^{2}+\xi_{r}^{2}+\left(\frac{\xi_{\phi}}{r}\right)^{2}}}\left[\xi_{z} R_{2}+\xi_{r} R_{3}+\frac{\xi_{\phi} R_{4}}{r}\right]+R_{1}
$$ 
where

$$
\begin{aligned}
& R_{1}=-\bar{v} p_{n}-\bar{w} p_{\xi}-\rho c^{2}\left(u_{n} n_{z}+v_{n} n_{r}+\frac{w_{n}}{r} n_{\phi}+u_{\zeta} \zeta_{z}+v_{\zeta} \zeta_{r}+\frac{w_{\zeta}}{r} \zeta_{\phi}\right) \\
& R_{2}=-\bar{v} u_{n}-\bar{w} u_{\zeta}-\frac{1}{\rho}\left(p_{n} n_{z}+p_{\zeta} \zeta_{z}\right) \\
& R_{3}=-\bar{v} v_{n}-\bar{w} v_{\zeta}-\frac{1}{\rho}\left(p_{n} n_{r}+p_{\zeta} \zeta_{r}\right) \\
& R_{4}=-\bar{v} w_{n}-\bar{w} w_{\zeta}-\frac{1}{\rho}\left(p_{n} \frac{n_{\phi}}{r}+p_{\zeta} \frac{\zeta_{\phi}}{r}\right)
\end{aligned}
$$

With $p$ specified as a constant in time, $p_{\tau}=0$, Eq. (15) is solved for $u_{\tau}$

$$
\begin{aligned}
& u_{\tau}=-\left[U+c \sqrt{\xi_{z}^{2}+\xi_{r}^{2}+\left(\frac{\xi_{\phi}}{r}\right)^{2}}\right] u_{\xi}+R_{2}+\frac{\xi_{r}}{\xi_{z}} R_{3}+\frac{\xi_{\phi}}{\xi_{z}} \frac{R_{4}}{r}+\frac{R_{1}}{\xi_{z}} \\
& -\frac{\xi_{r}}{\xi_{z}}\left\{v_{\tau}+\left[U+c \sqrt{\xi_{z}^{2}+\xi_{r}^{2}+\left(\frac{\xi_{\phi}}{r}\right)^{2}}\right] v_{\xi}\right\} \\
& -\frac{\xi_{\phi}}{r \xi_{z}}\left\{w_{\tau}+\left[u+c \sqrt{\xi_{z}^{2}+\xi_{r}^{2}+\left(\frac{\xi_{\phi}}{r}\right)^{2}}\right] w_{\xi}\right\} \\
& -\frac{\sqrt{\xi_{z}^{2}+\xi_{r}^{2}+\left(\frac{\xi_{\phi}}{r}\right)^{2}}}{\rho C \xi_{z}} \quad\left\{\left[U+c \sqrt{\xi_{z}^{2}+\xi_{r}^{2}+\left(\frac{\xi_{\phi}}{r}\right)^{2}}\right] p_{\xi}\right\}
\end{aligned}
$$

For the grid being used for this problem, $\xi_{r}=\xi_{d}=0$ at the down-stream boundary, causing a simplification of Eq. (16). If this was not the case, Eq. (16) would have to be solved simultaneously with the v-and w-momentum equation.

Writing equations with the simplification, $\xi_{r}=\xi \phi=0$, the following equation for the u-velocity is obtained.

$$
u_{\tau}=-\left(U+c \xi_{z}\right) u_{\xi}+R_{2}+\frac{R_{1}}{\xi_{z}}-\frac{\xi_{z} p_{\xi}}{\rho}
$$




\section{INITIAL CONDITIONS}

The solution procedure can be started from specified freestream conditions. At time $t=0$, the values of the dependent variables are set equal to freestream conditions at every node of the mesh. At the first time step tangency conditions are instantaneously applied in the manner previously described. No startup problems have been encountered with this approach.

An alternate approach is to start from a previously calculated solution. If different free stream conditions are specified, the upstream and radial far field conditions are changed and the solution procedure is restarted. If a different mesh is being used, the dependent variable vector $Q$ must be rescaled by the new Jacobian to avoid start up problems.

\section{NUMER ICAL RESULTS}

This analysis has been used to calculate the flow around propellers operating at high subsonic speeds. Results will be shown for the eight bladed SR-3 propeller (ref. 14) shown in figure 2. The grid used for these calculations is shown in figure 3 . Figure $3(a)$ shows the grid along one surface of a blade (shaded region) and a portion of the grid in the vicinity of the blade at a constant value of the index in the circumferential direction.

Figure $3(b)$ shows the grid between two blades at a constant value of the index in the radial direction and in figure $3(\mathrm{c})$ at a constant value of the index in the axial direction. A total of 45 points in the "axial" direction, 21 points in the "radial" direction and 11 points between adjacent blades in the "circumferential" direction were used for the calculations to be described. on the blade 21 points in the chordwise direction and 12 points in the spanwise direction were used.

Blade geometry used in the calculations includes deformation due to centrifugal loads during operation. Blade deformation was calculated using a finite element structural analysis program at the design condition of the propeller: free stream Mach number of 0.8 , advance ratio of 3.06 and blade angle at three-quarter radius of $57^{\circ}$. The same deflection was used for all blade angles at the design rotational speed. At other rotational speeds the deflection was scaled by the square of the rotational speed.

Flow properties on the blade surfaces of the SR-3 propeller at a free stream Mach number of 0.6 are shown in figures 4 and 5 . The blade angle at a radial location equal to three-quarters of the tip radius is $60.5^{\circ}$ and the advance ratio is 3.06 . Isobars on the suction and pressure surfaces of the blades are shown in figure 4. Regions of constant relative Mach number are shown in figure 5 . The generally increasing level of relative Mach number from the nacelle surface toward the tip is a result of the increasing rotational speed with increasing radius. Each shade of gray indicates a range of 0.1 in both static pressure ratio and relative Mach number. Both the static pressure, figure $4(\mathrm{a})$, and relative Mach number distribution, figure $5(\mathrm{a})$, on the suction side of the blade indicate higher load on the forward portion of the blade. Near the nacelle surface on the suction side, the high pressure and low Mach number regions at about the quarter chord location are caused by 
the rapid increase in spinner diameter at that location. On the pressure side of the blade, figure 5(b), the high relative Mach numbers at about mid-chord, which is the location of maximum thickness on the blades, is apparently related to the thickness distribution of the airfoils used in the blade.

Computed values of swirl angle downstream of the propeller are shown in figure 6. Also shown are experimentally measured values of swirl angle obtained during the wind tunnel tests described in reference 14 . These values were measured with an instrumented wedge mounted on a translating probe figure 2. These probe results have not been previously reported. Both the computed and measured values correspond to an axial location 0.21 diameters downstream of the pitch change axis. Although the level of the predicted results is considerably higher than the experimental results, the radial variation of swirl is in reasonable agreement between the two sets of results. An estimate of viscous effects has been made using the compressor methodology of reference 15. At a radius ratio $r / R=0.52$ which corresponds to a solidity (chord/gap) of 1.0 , the deviation angle was determined to be $3.15^{\circ}$. This corresponds to a reduction of the swirl angle to a value of $5.8^{\circ}$ from the originally predicted $8.9^{\circ}$. It thus appears that at lower free stream Mach numbers the overprediction of power coefficient is due to neglecting viscous effects in the analysis and that boundary layer growth on the blade surface causes a reduction in swirl angle downstream of the propeller. The unusual behavior of the data near $r / R=1.0$ is apparently caused by the tip vortex roll up. The analysis has not predicted this feature of the flow because the mesh is too coarse in this region.

A comparison of computed and measured propeller power coefficient is shown in figure 7. Experimental results are taken from reference 14 . The power coefficient is considerably overpredicted. Since power coefficient is closely related to swirl angle the overprediction of power coefficient is consistent with the overprediction of swirl angle shown in figure 6 .

Predicted blade surface flow properties for the SR-3 propeller at a free stream Mach number of 0.8 are shown in figures 7 and 8 . The blade angle at three-quarter radius is $61.3^{\circ}$ and the advance ratio is 3.06 . The pressure distribution on the suction side of the blade is shown in figure $8(a)$. Near the nacelle surface the low pressure region at about one quarter chord is caused by the rapid increase in spinner diameter at that location. Just downstream a second lower pressure region is observed followed by a rapid change to high pressure suggests the presence of a shock wave at about two-thirds chord. With increasing distance from nacelle to tip the strength of this compression decreases and moves to the trailing edge of the blade. On the pressure side of the blade, figure $8(b)$, the rapid compression near the nacelle surface at about two-thirds chord moves forward with increasing distance from nacelle to tip.

The relative Mach number distribution on the suction side of the blade, figure $9(a)$, generally follows the same trends as the pressure distribution of figure $8(a)$. The level of Mach number near the nacelle surface at about two-thirds chord indicates the presence of a shock wave at that location. On the pressure side of the blade, figure 9(b), a shock wave is indicated at about the same location. Whereas the lines of constant pressure and constant relative Mach number on the suction side of the blade have similar shapes, they are somewhat different on the pressure side. The reason for this difference is unknown at this time. 
The static pressure distribution in the flow field between two adjacent blades is shown in figure 10. The suction surface is at the bottom of the plots and the pressure surface at the top. At the nacelle surface, figure 10 (a), the shock wave seen in figures 8 and 9 on both sides of the blades can clearly be seen spanning the passage between the blades. A small expansion region can be seen near the leading edge of the blade on the suction surface. The static pressure distribution on a mesh surface which has $r / R=0.82$ at the mid chord is shown in figure 10(b). The shock wave from the trailing edge of the blade on the suction surface spans the blade passage and intersects the pressure side of the adjacent blade.

Relative Mach numbers in the flow field between two blades at a free stream Mach number of 0.8 are shown in figure 11 . General features are simi1 ar to the pressure contours shown in figure 10. The main difference is near the pressure surface in figure 11(b) where a rise in Mach number is observed without a corresponding drop in pressure in figure 10(b). The reason for this is not known. The results shown in figure $11(b)$ are in very good qualitative agreement with laser velocimeter measurements presented in reference 16 . A significant difference is the location of the shock wave. In the computed results the shock wave originates at the trailing edge of the blade on the suction surface whereas the data of reference 16 indicates a shock wave location somewhat upstream of the trailing edge. This behavior is consistent with shock wave boundary layer interactions in other types of flow fields in which viscous effects cause the actual shock wave location to be upstream of the location predicted by inviscid analyses.

Predicted and measured swirl angle downstream of the propeller at a free stream Mach number of 0.8 are shown in figure 12. Numerical results for swirl angle are higher than those measured in the wind tunnel (ref. 14) similar to the results at a Mach number of 0.6 (fig. 6). The observed shock wave location upstream of the trailing edge would result in reduced blade loading causing reduced swirl relative to a trailing edge shock wave location. This would at least partially explain the overprediction of swirl angle shown in figure 12. This overprediction is approximately equal to five degrees whereas at a free stream Mach number of 0.6 the discrepancy is about three degrees. This implies that at free stream Mach number of 0.8 a mechanism in addition to boundary layer growth is causing decreased flow turning. This mechanism could be the shock wave boundary layer interaction discussed previously. The compressor methodology of reference 15 cannot be used to estimate viscous losses at this condition. This methodology is based on low speed cascade data and the presence of shock waves in the flow field clearly makes it not applicable. The radial variation of swirl angle agrees reasonably well with the experimental results. The region with the largest discrepancy is again the tip region where the coarse mesh cannot resolve the details of the flow associated with the tip vortex.

The general features of the tip vortex, however, are predicted by the analysis as shown in figure 13. The secondary flow velocity vectors on a mesh surface just downstream of the propeller at a free stream Mach number of 0.8 as predicted by the analysis are shown. These vectors are composed of the radial and circumferential components of the flow field velocities. Two blade passages are shown with a blade at each edge of the region shown and a third blade approximately in the middle. The tip vortex can clearly be seen as well as the jump in spanwise component of velocity in the inboard region associated with increasing loading with radial distance. 
A summary of propeller power coefficient for the SR-3 propeller at a free stream Mach number of 0.8 is shown in figure 14. The SR-3 propeller was designed for a cruise condition at a free stream Mach number of 0.8 , advance ratio of 3.06 and a power coefficient of 1.7. Power coefficient is shown for three blade angle settings. Although the power coefficient is again overpredicted, the variation of power coefficient with blade angle is predicted quite well. The overprediction of power coefficient is consistent with the overprediction of swirl angle shown in figure 12 and would improve if shock wave boundary layer interaction effects were included.

\section{CONCLUSIONS}

A computer program, NASPROP-E, has been written to calculate the flow field around high speed propellers. This program has been applied to a propeller with eight highly swept blades and an axisymmetric nacelle operating at transonic conditions. Numerical results indicate the presence of shock waves in the flow field and the roll up of the tip vortex.

Comparisons of numerical results with data indicate the following:

1. Propeller power coefficient and swirl angle downstream of the propeller are overpredicted. At lower free stream Mach numbers this discrepancy can be attributed to viscous effects. At higher free stream Mach numbers this discrepancy is apparently due to shock wave boundary layer interaction effects.

2. Radial variation of swirl angle downstream of the propeller is predicted reasonably well. Details associated with the tip vortex are not as well defined due to a relatively coarse grid in that region.

3. The variation of propeller power coefficient with blade angle is predicted very well.

\section{REFERENCES}

1. Lindemuth, I., and Killeen, J., "Alternating Direction Implicit Techniques for Two-Dimensional Magnetohydrodynamic Calculations," Journal of Computational Physics, Vol. 13, 1973, pp. 181-208.

2. Briley, W.R., and McDonald, H., "Solution of the Multidimensional. Compressible Navier-Stokes Equations by a Generalized Implicit Method," Journal of Computational Physics, Wol. 24, Aug. 1977, pp. 372-397.

3. Briley, W.R., and McDonald, H., "Solution of the Three-Dimensional Compressible Navier-Stokes Equations by an Implicit Technique," Proceedings of the Fourth International Conference on Numerical Methods in Fluid Dynamics, edited by R.D. Richtmyer, Springer-Verlag, Berlin, 1974, pp. 105-110.

4. Beam, M., and Warming, R.F., "An Implicit Finite-Difference Algorithm for Hyperbolic Systems in Conservation-Law Form," Journal of Computational Physics, Vol. 22, 1976, pp. 87-110.

5. Beam, R., and Warming, R.F., "An Implicit Factored Scheme for the Compressible Navier-Stokes Equations," AIAA Journal, Vol. 16, No. 4, Apr. 1978, pp. 393-402. 
6. Steger, J.L. and Kutler, P., "Implicit Finite-Difference Procedures for the Computation of Vortex Wakes," AIAA Journal, Vol. 15, No. 4, April 1977 , pp. 581-590.

7. Kutler, P., Chaussee, D., and Pulliam, T., "Supersonic Flow over Ablated Nosetips Using an Implicit Unsteady Euler Equation Solver, " Paper presented at the Open Forum Session of the AIAA 10th Fluid and Plasma Dynamics Conference, Albuquerque, New Mexico, June 1977.

8. Stenger, J.L., "Implicit Finite-Difference Simulation of Flow about Arbitrary Geometries with Application to Airfoils," AIAA Paper 77-665, June 1977.

9. Pulliam, T.H. and Steger, J.L., "Implicit Finite-Difference Simulations of Three-Dimensional Compressible Flow, " AIAA Journal, Vol. 18 No. 2, Feb. 1980, pp. 159-167.

10. Warming, R.F. and Beam, R.M., "On the Construction and Application of Implicit Factored Schemes for Conservation Laws, " Computational Fluid Dynamics, H.B. Keller, American Mathematical Society, Providence, 1978, pp. 85-129.

11. Pulliam, T.H., and Chaussee, D.S., "A Diagonal Form of an Implicit Approximate-Factorization Algorithm, "Journal of Computational Physics, Vol. 39, No. 2, Feb. 1981, pp. 347-363.

12. Isaacson, E. and Keller, H.B., Analysis of Numerical Methods, John Wiley and Sons, Inc., New York, 1969.

13. Chaussee, D.S., and Kutler, P., "User's Manual for Three-Dimensional Analysis of Propeller Flow Fields," NASA CR-167959; Dec. 1982.

14. Rohrbach, C., Metzger, F.B., Black, D.M., and Ladden, R.M., "Evaluation of. Wind Tunnel Performance Testings of an Advanced $45^{\circ}$ Swept Eight-Bladed Propeller at Mach Numbers from 0.45 to 0.85," NASA CR-3505, Mar. 1982.

15. Johnsen, I.A. and Bullock, R.0., eds Aerodynamic Design of Axial-Flow Compressors, NASA SP-36, 1965.

16. Neumann, H.E., Bober, L.J., Serafini, J.S., and Chang, L.K., "An Analytical and Experimental Comparison of the Flow Field of an Advanced Swept Turboprop," AIAA Paper No. 83-0189, Jan. 1983. 


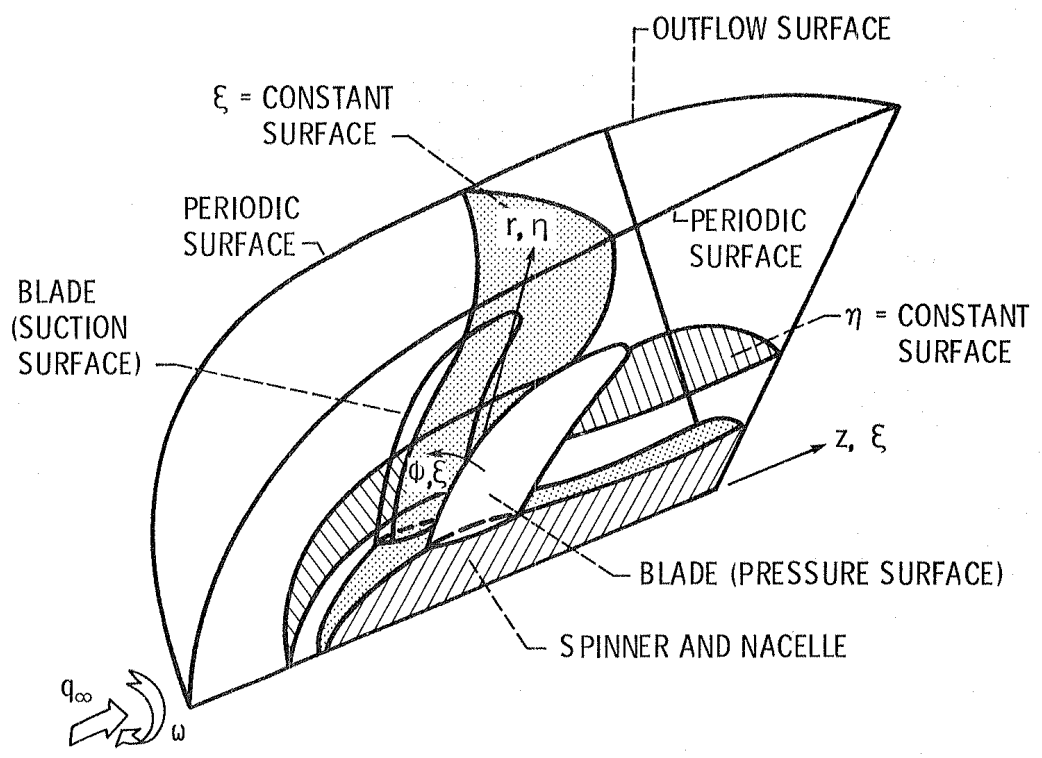

Figure 1. - Coordinate system.

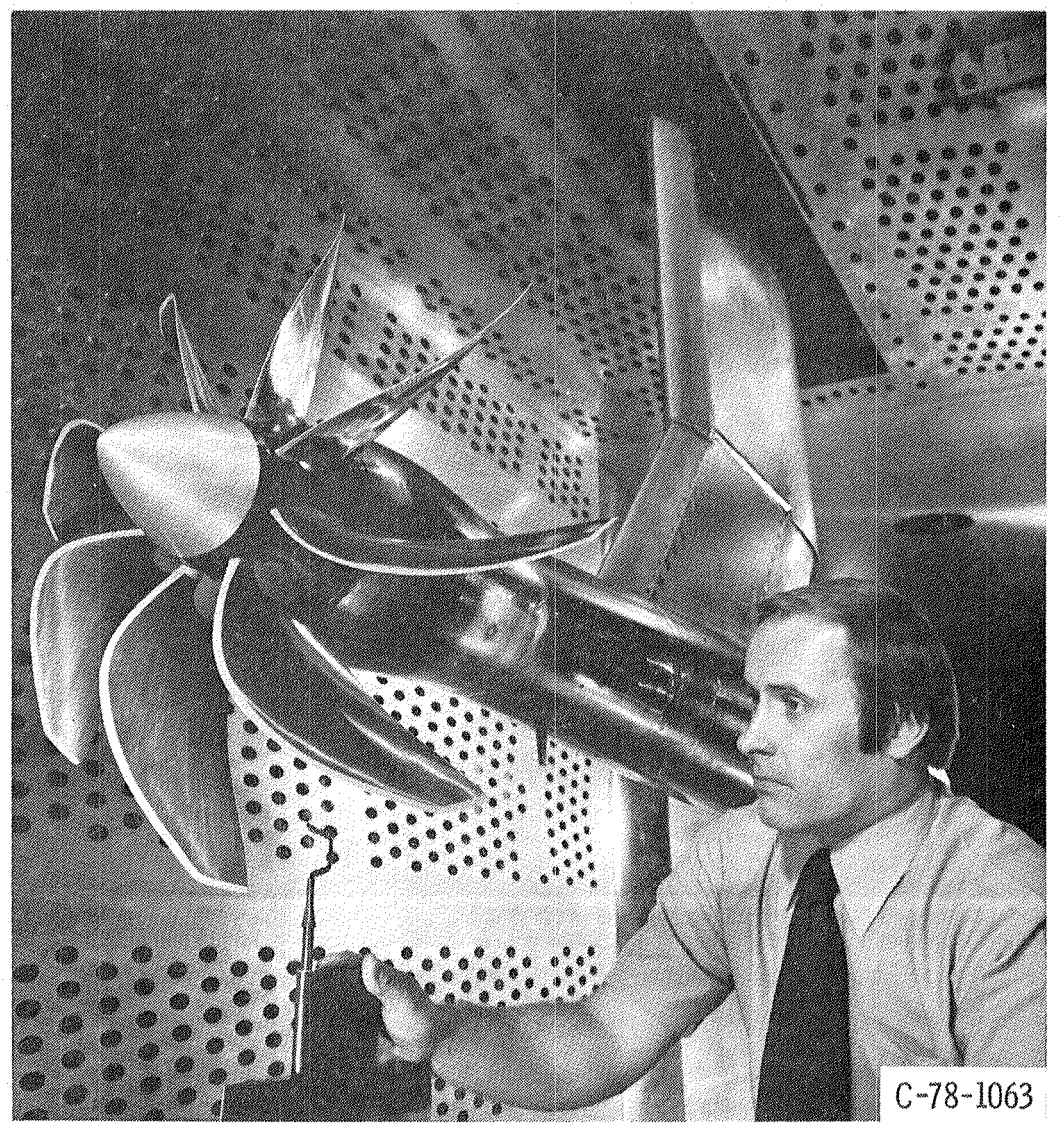

Figure 2. - SR-3 propeller in the NASA-Lewis Research Center 8x6 supersonic wind tunnel. 


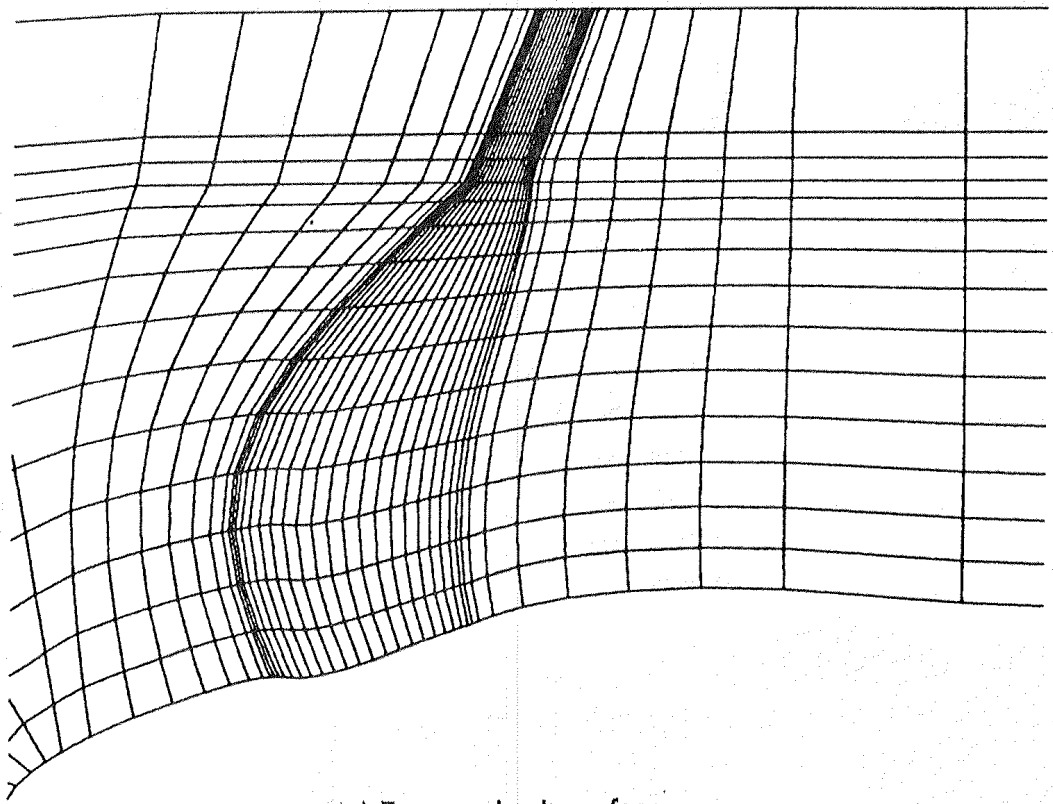

(a) $\zeta=$ constant surface.

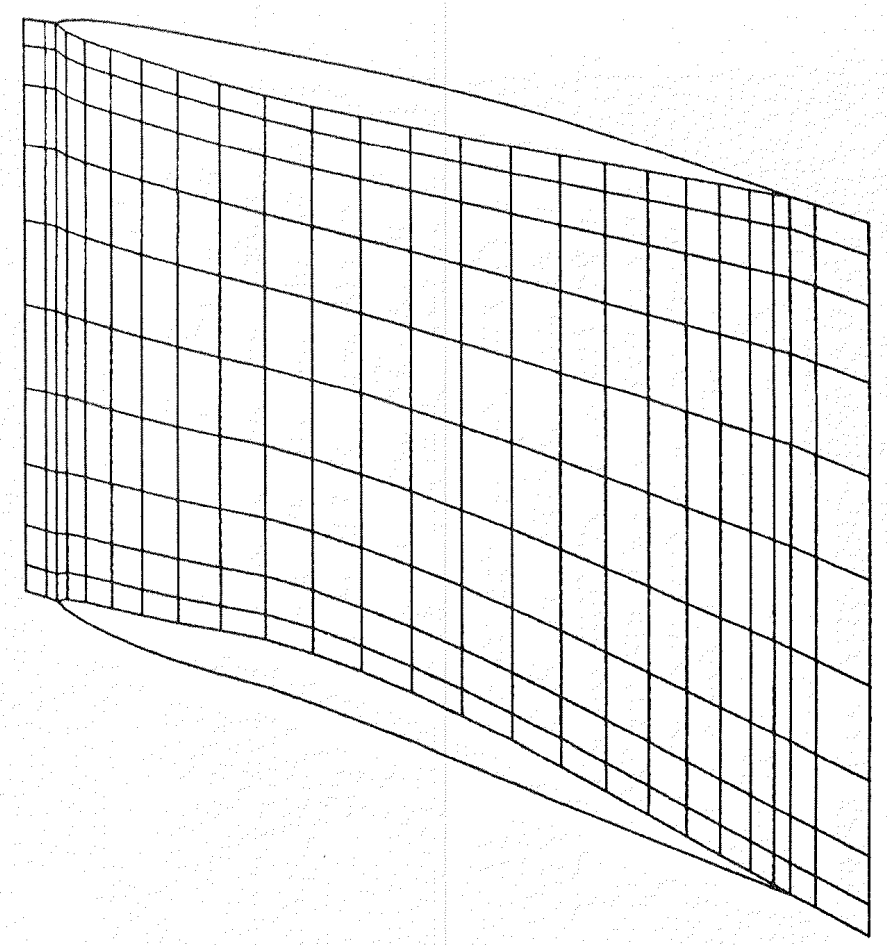

(b) $\eta=$ constant surface.

Figure 3. - Mesh in the vicinity of the blades used for calculation of the flow around the SR-3 propeller. 


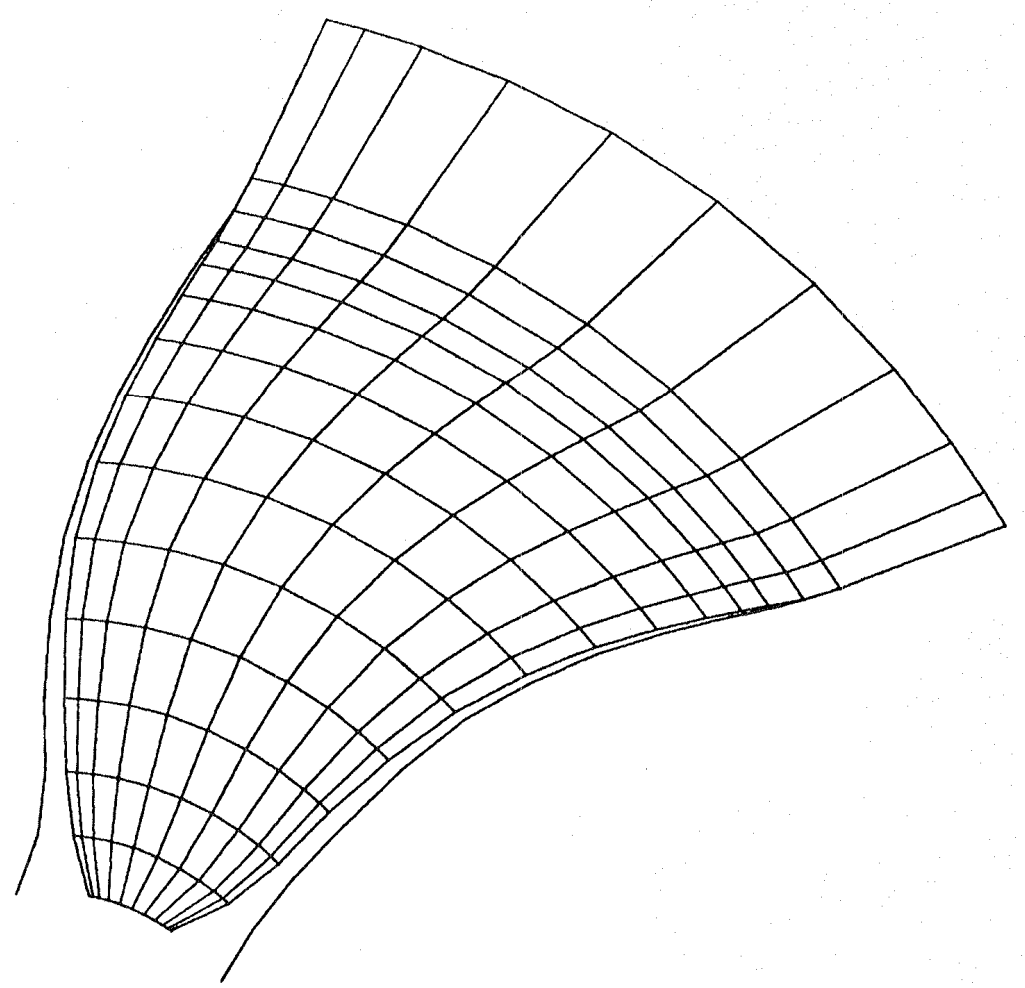

(c) $\xi=$ constant surface.

Figure 3. - Concluded. 




(a) Suction side.

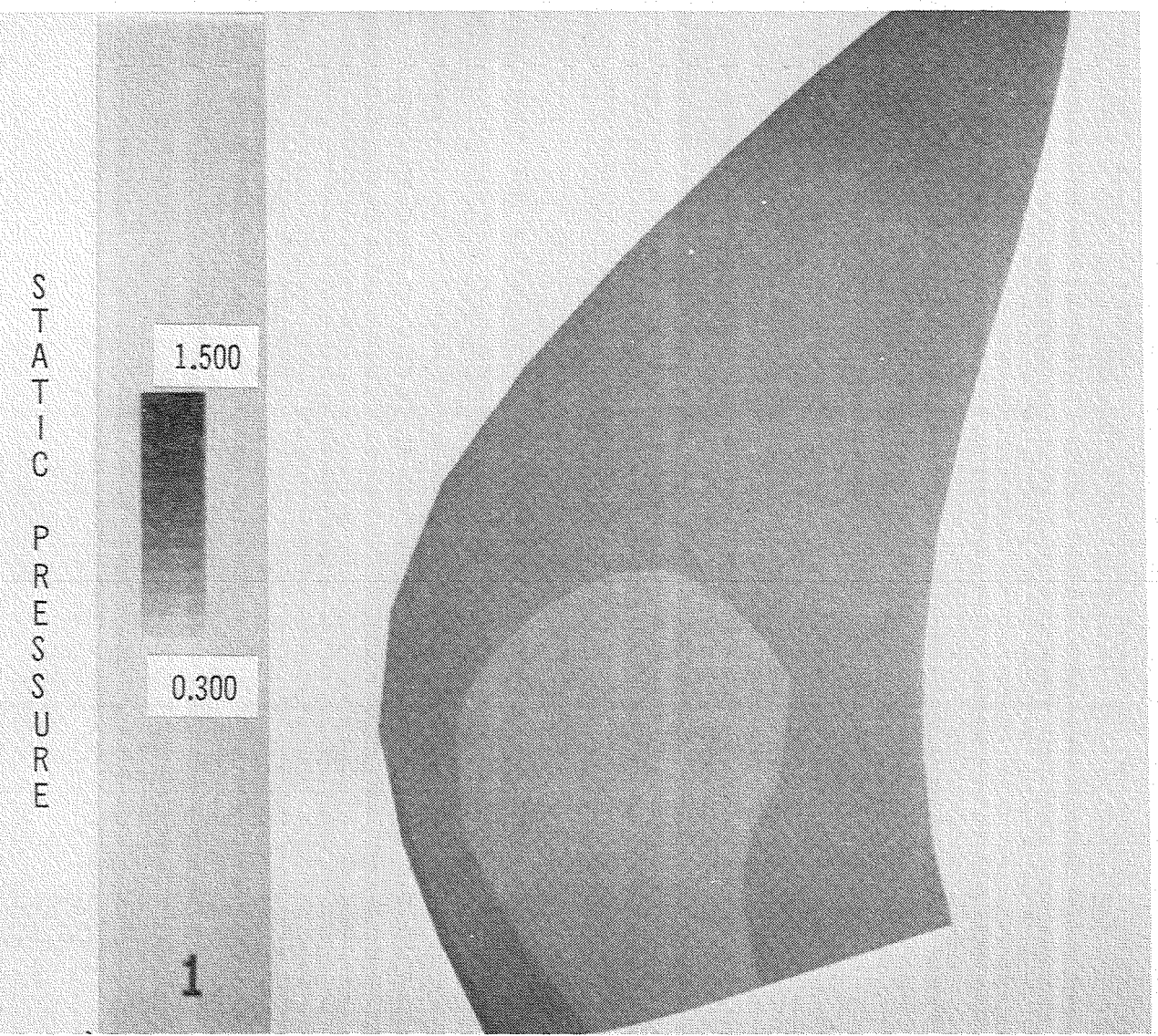

(b) Pressure side.

Figure 4. - Predicted static pressure distribution, $\mathrm{p} / \mathrm{p}_{\infty}$, on both sides of the SR-3 propeller blade. Free stream Mach number, 0.6 ; advance ratio, 3.06; blade angle at three-quarter radius, $60.5^{\circ}$. 


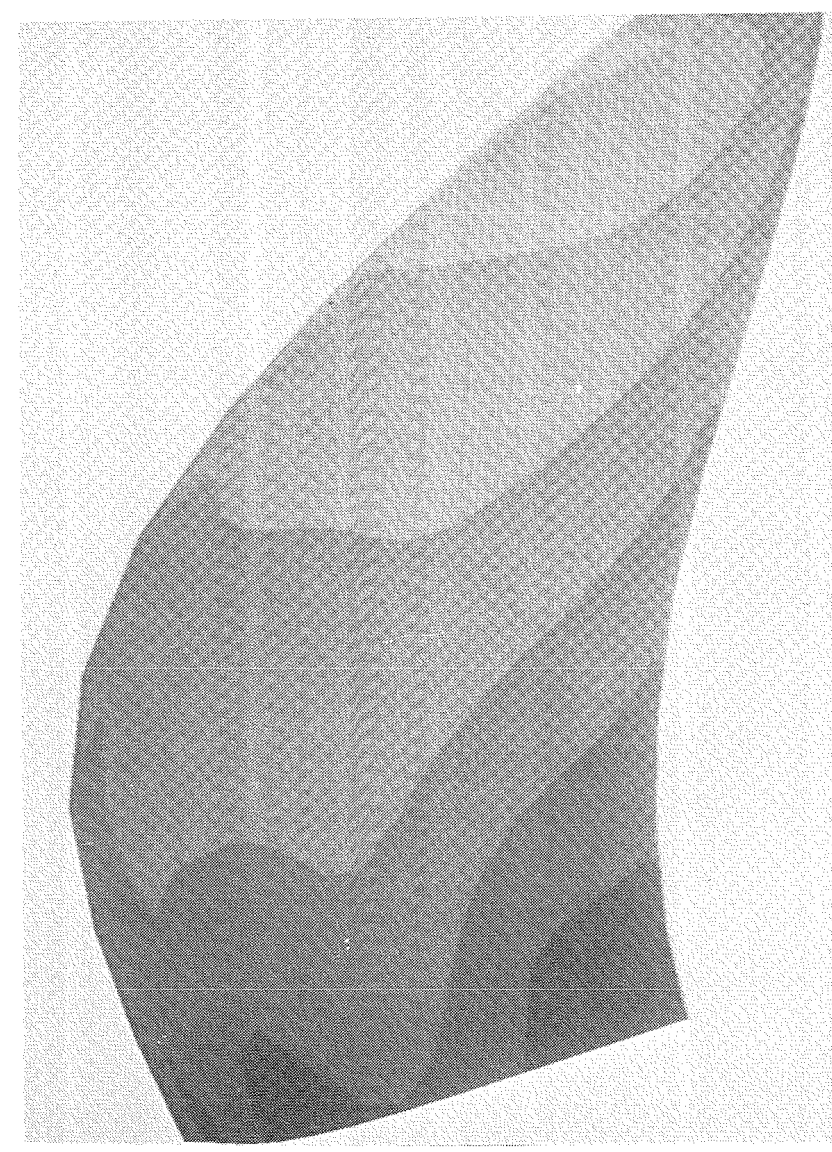

(a) Suction side

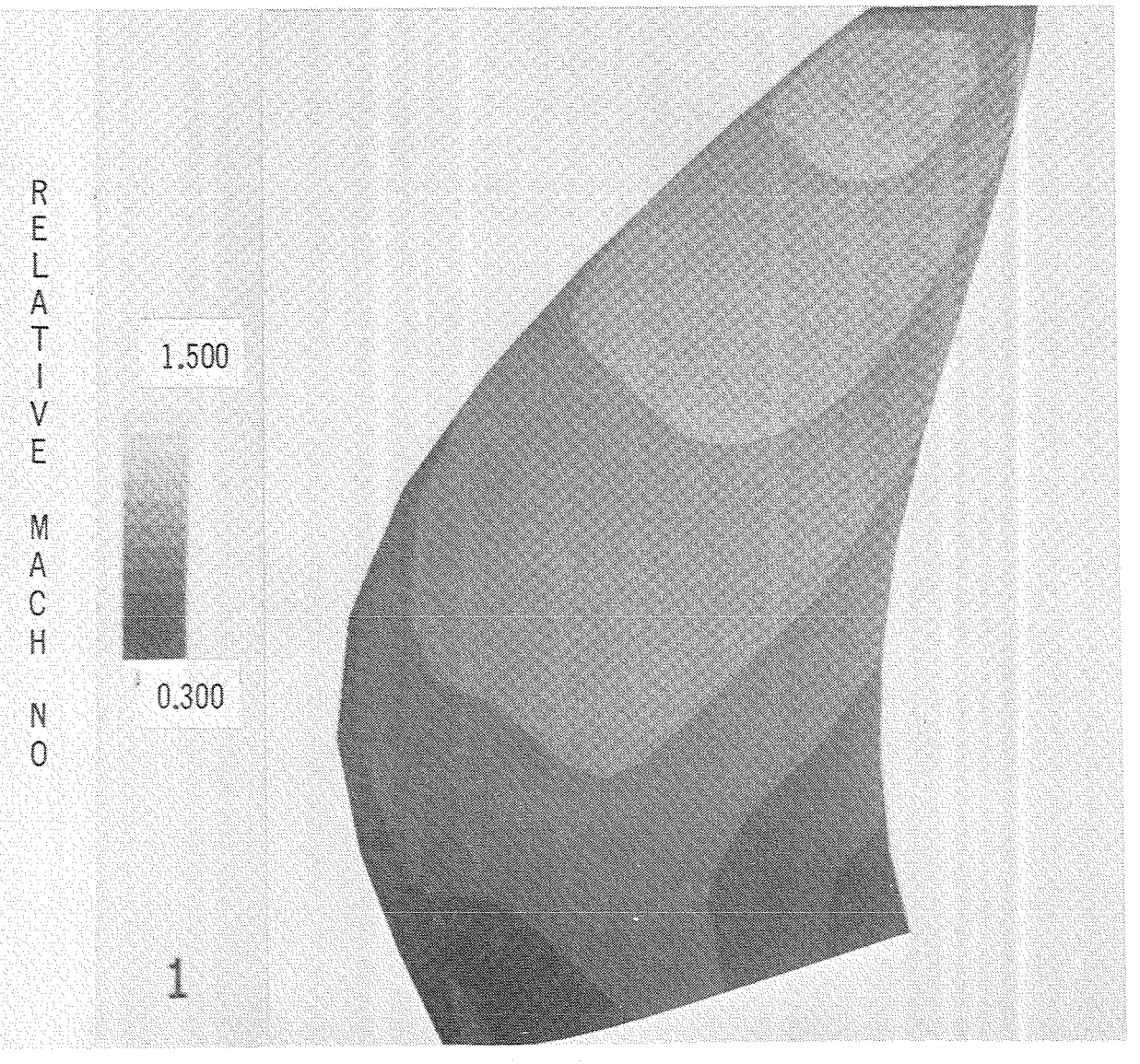

(b) Pressure side.

Figure 5. - Predicted distribution of relative Mach number on both sides of the SR-3 propeller blade. Free stream Mach number, 0.6; advance ratio, 3.06; blade angle at three-quarter radius, $60.5^{\circ}$. 


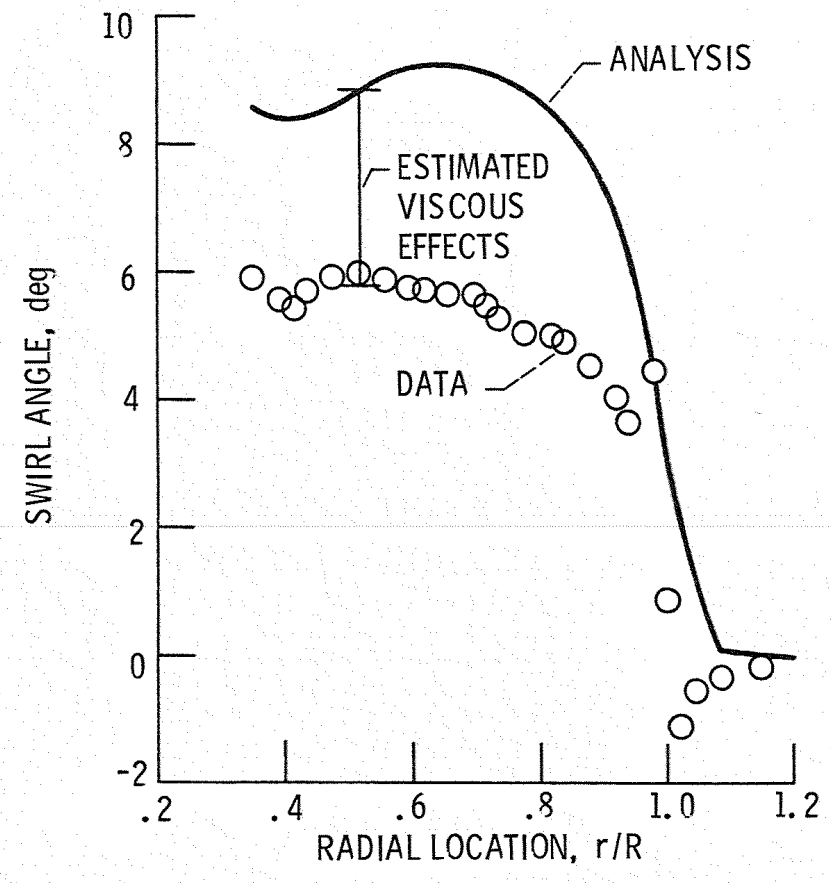

Figure 6. - Comparison of predicted and measured swirl angle downstream of the SR-3. propeller blade. Axial location, 0.21 diameters downstream of the pitch change axis; free stream Mach number, 0.6; advance ratio, 3.06; blade angle at three-quarter radius, $60.5^{\circ}$.

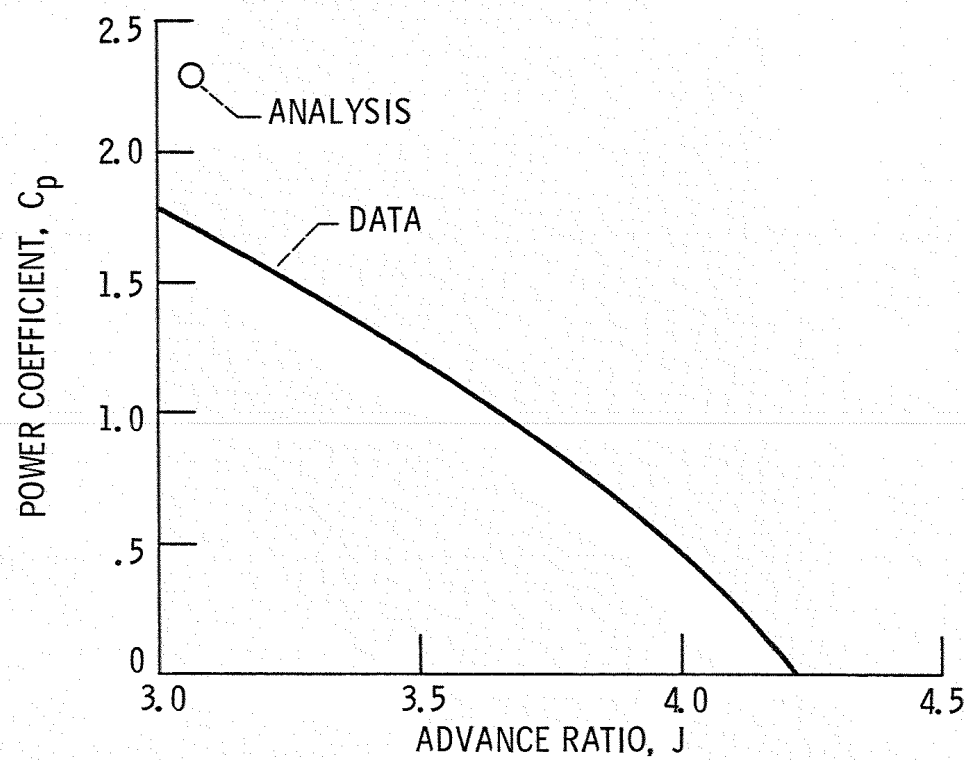

Figure 7. - Comparison of predicted and measured power coefficient for the SR-3 propeller. Free stream, Mach number, 0.6; advance ratio, 3.06; blade angle at three-quarter radius, $60.5^{\circ}$. 


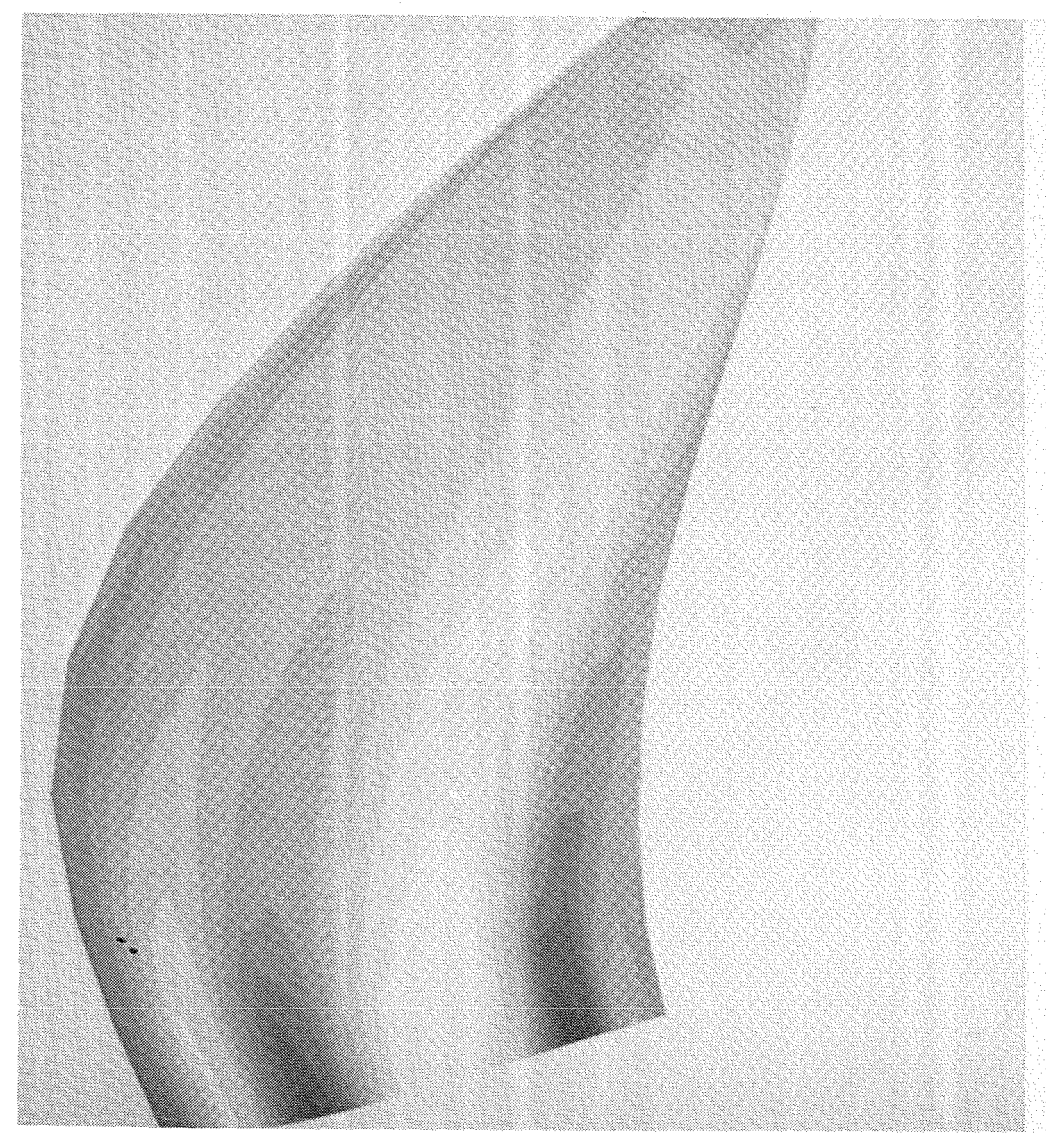

(a) Suction side.

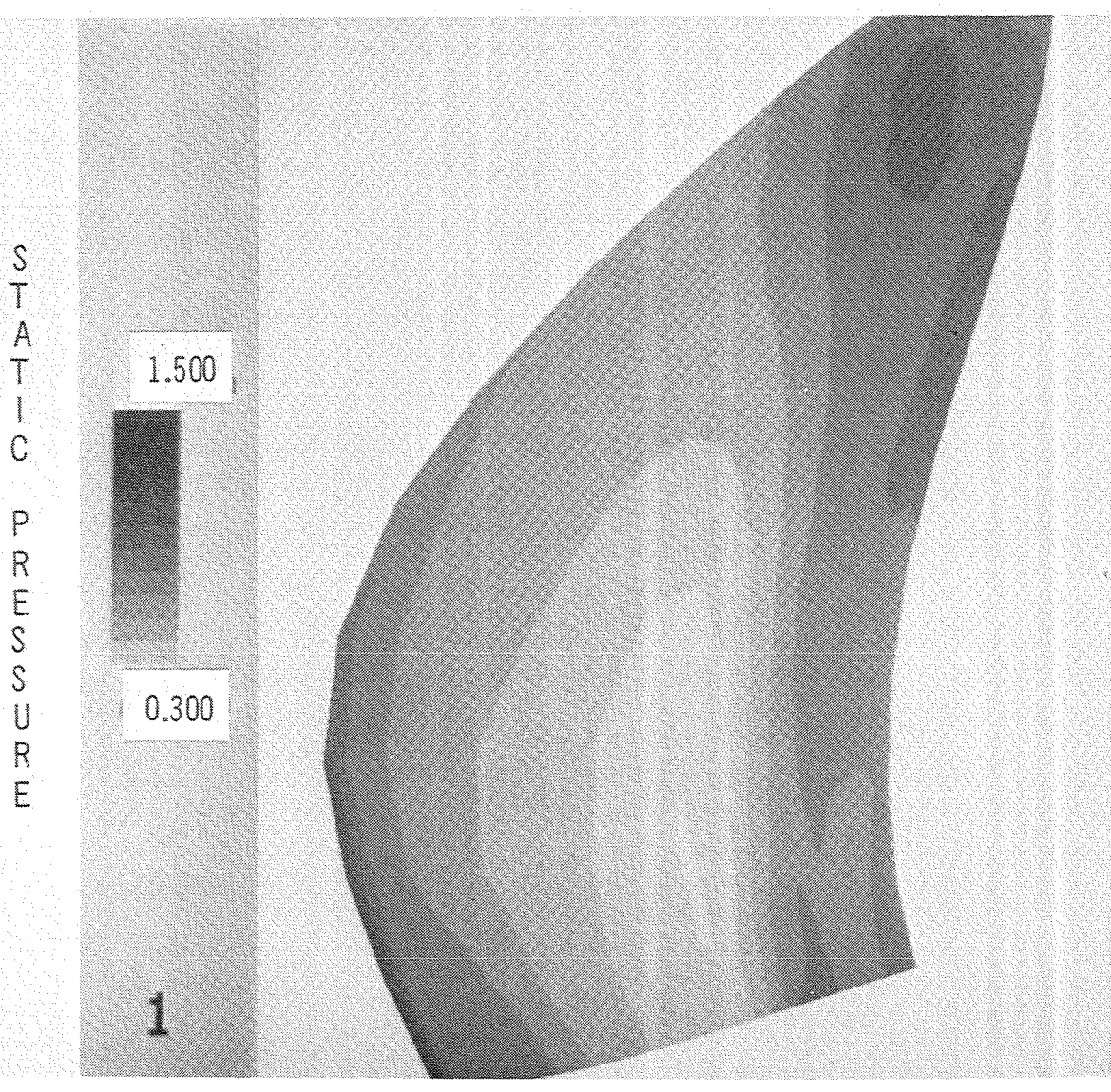

(b) Pressure side.

Figure 8. - Predicted static pressure distribution, $\mathrm{p} / \mathrm{p}_{\infty}$, on both sides of the SR-3 propeller blade. Free stream Mach number, 0.8 ; advance ratio, 3.06; blade angle at three-quarter radius, $61.3^{\circ}$. 


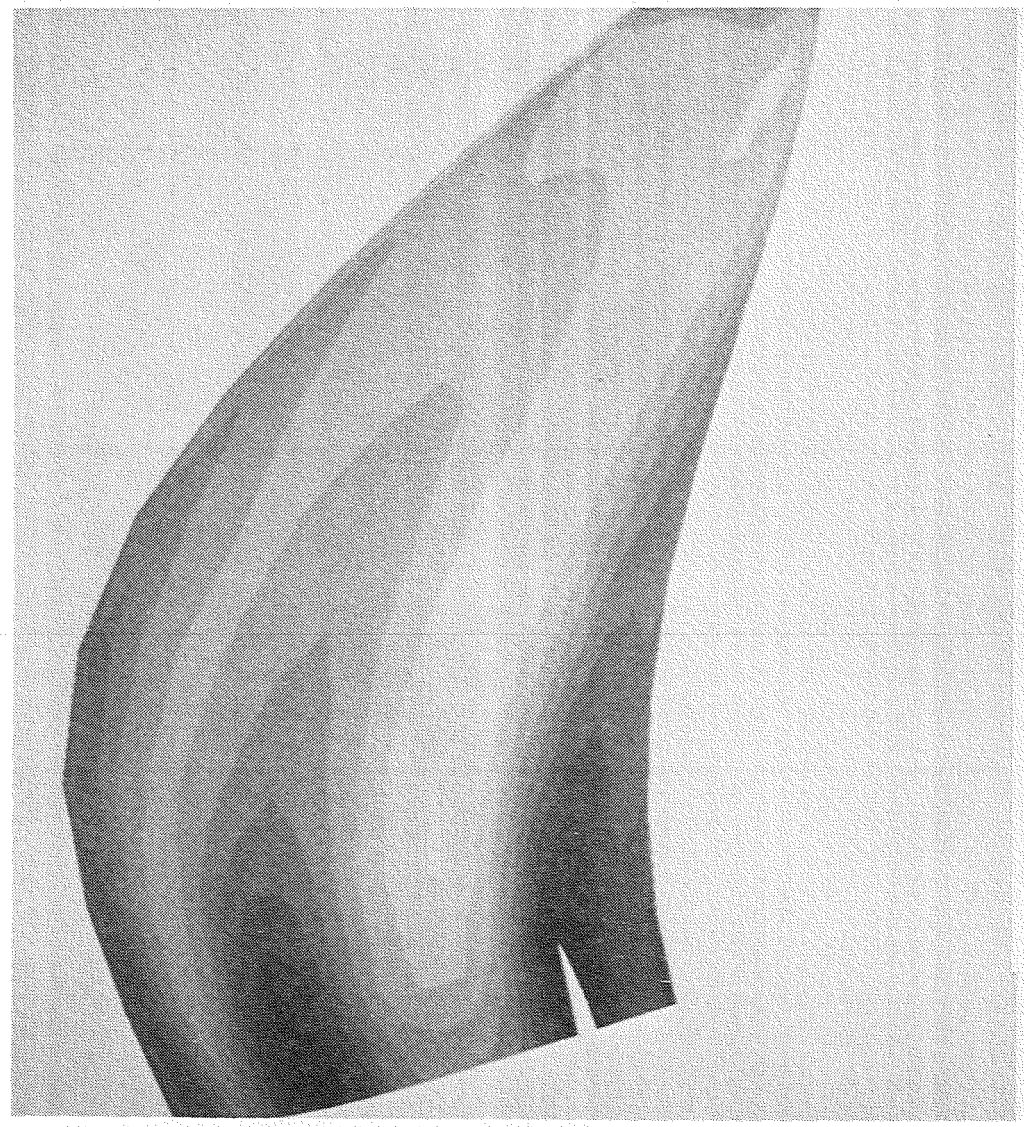

(a) Suction side.

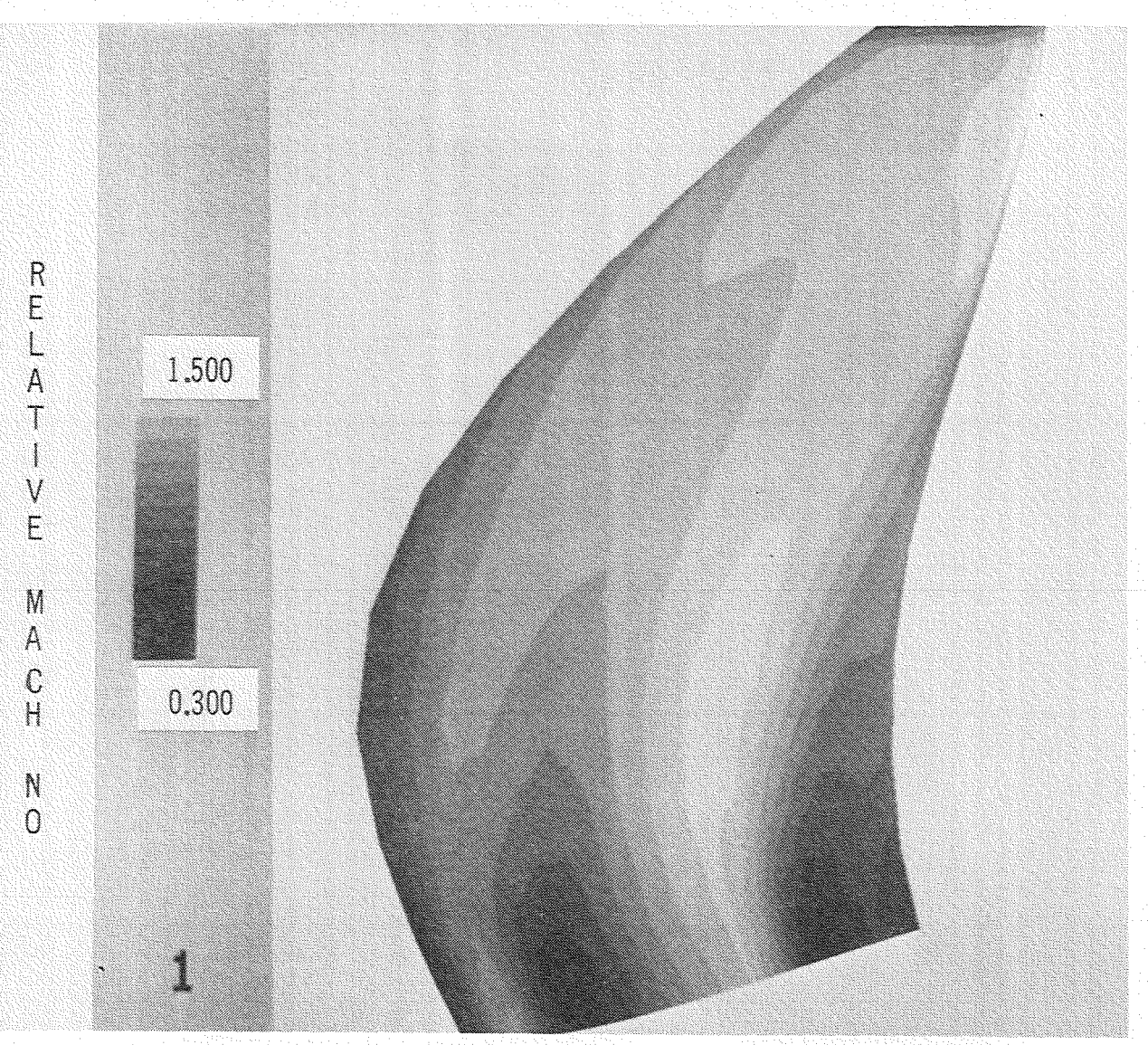

(b) Pressure side.

Figure 9. - Predicted distribution of relative Mach number on both sides of the SR-3 propeller blade. Free stream Mach number, 0.8; advance ratio, 3.06; blade angle at three-quarter radius, $61.3^{\circ}$. 


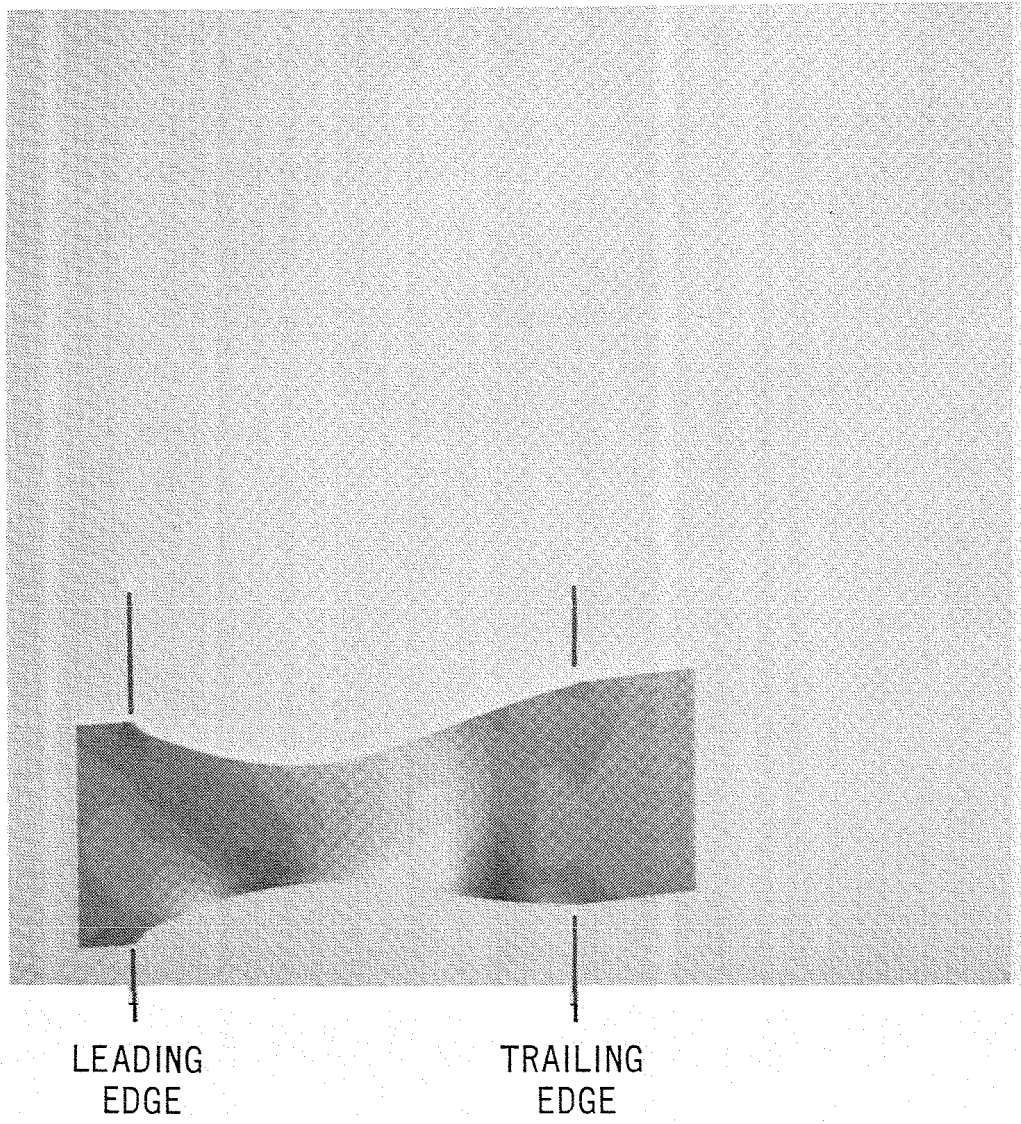

(a) $r / R=0.24$ (nacelle surface).

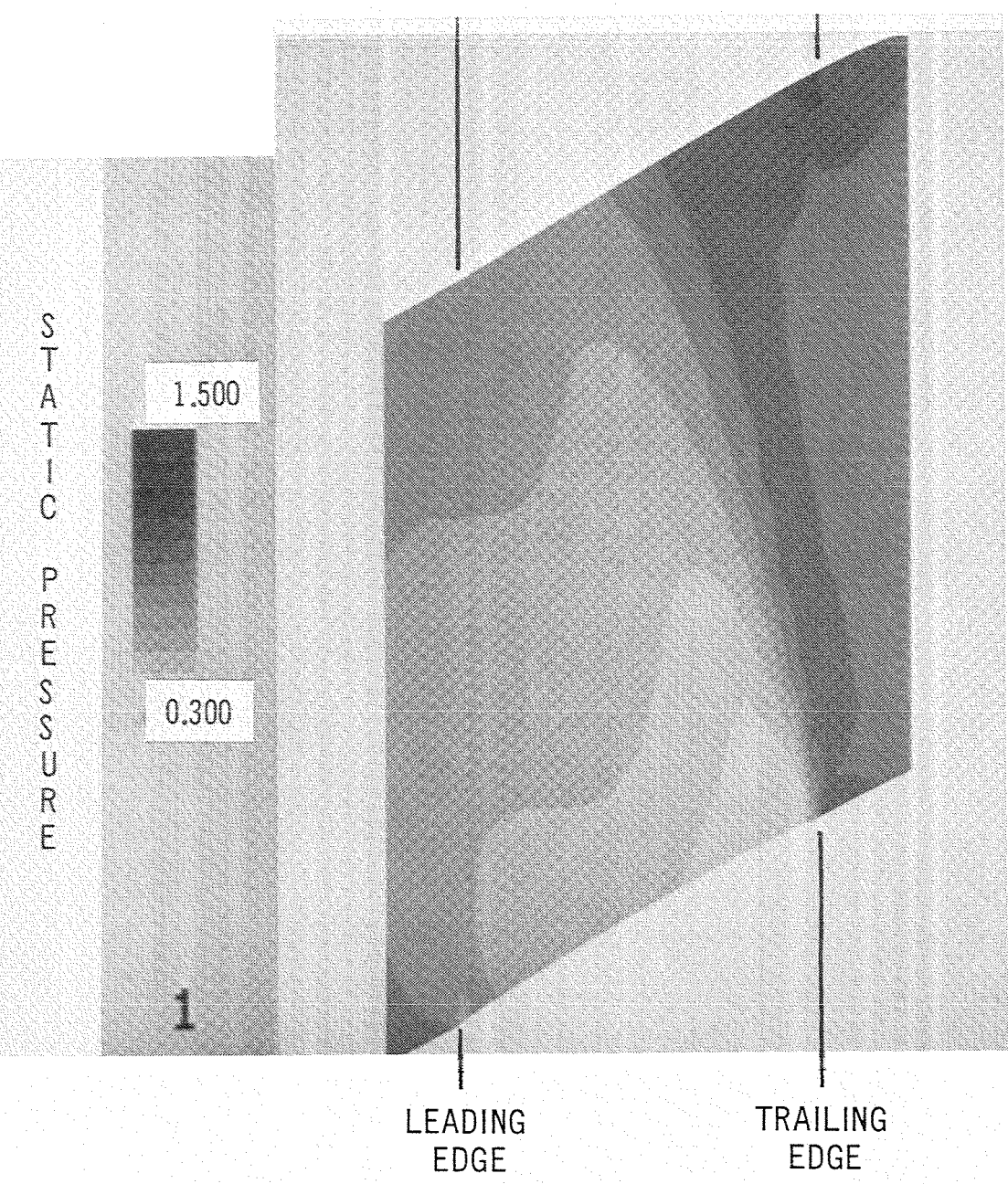

(b) $r / R=0.82$.

Figure 10. - Predicted static pressure distribution, $\mathrm{p} / \mathrm{p}_{\infty}$, between the blades of the SR-3 propeller. Free stream Mach number, 0.8; advance ratio, 3.06; blade angle at three-quarter radius, $61.3^{\circ}$. 


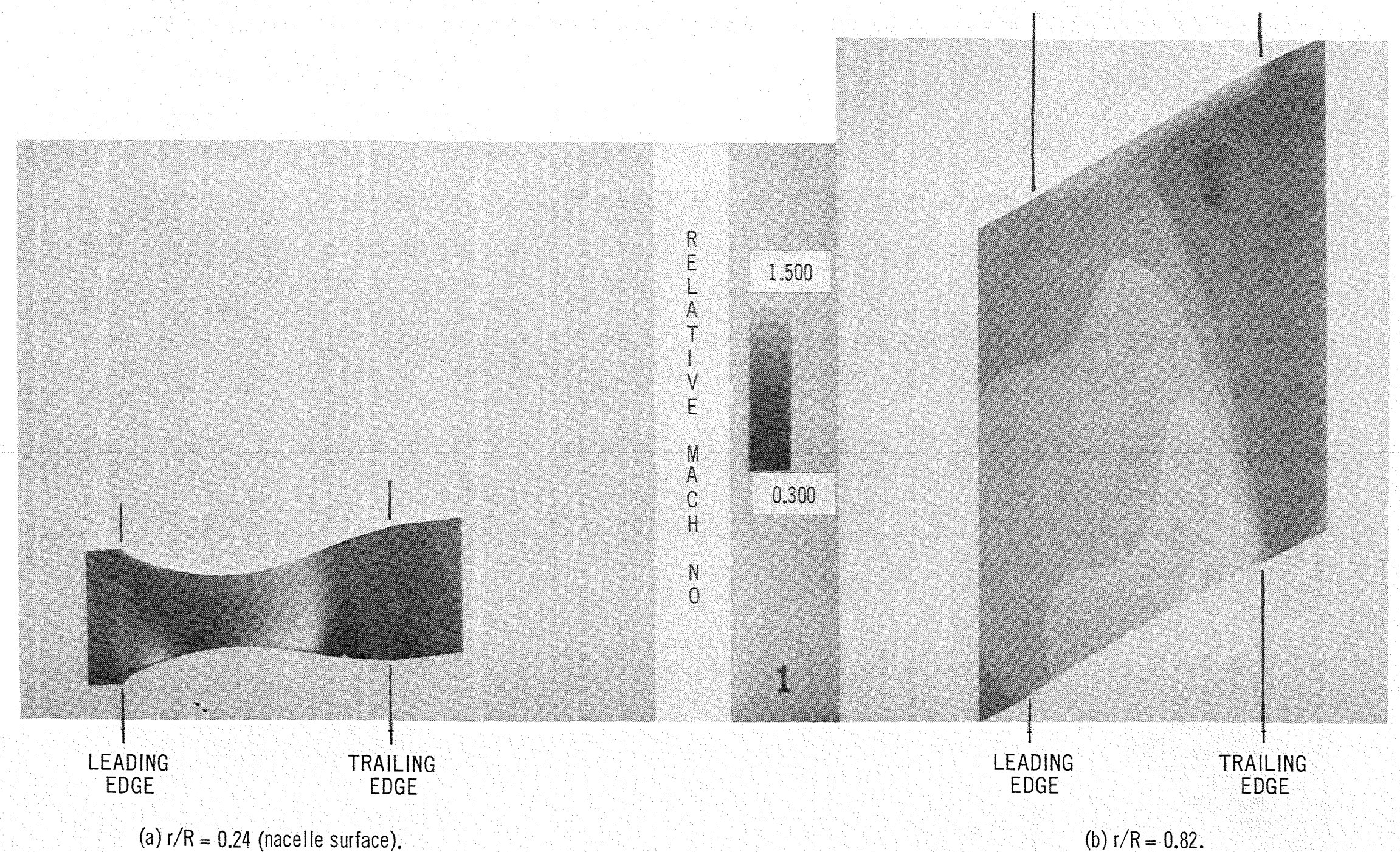

Figure 11. - Predicted distribution of relative Mach number between the blades of the SR-3 propeller. Free stream Mach number, 0.8 ; advance ratio, 3.06; blade angle at three-quarter radius, $61.3^{\circ}$. 


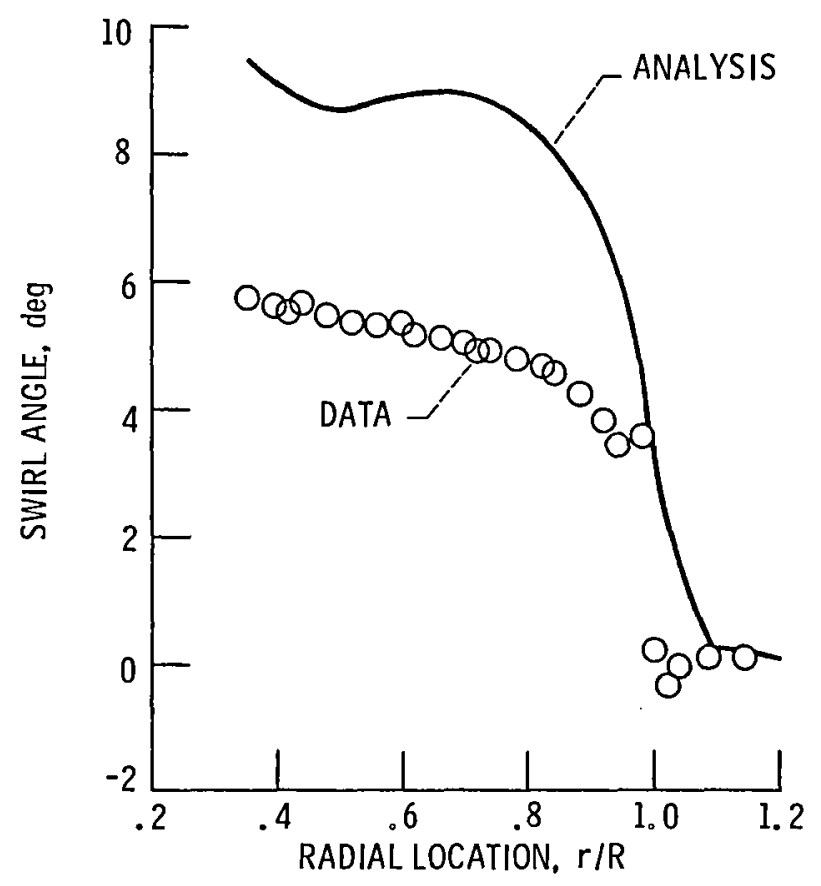

Figure 12. - Comparison of predicted and measured swirl angle downstream of the SR-3 propeller blade. Axial location, 0.21 diameters downstream of the pitch change axis; free stream Mach number, 0.8; advance ratio, 3.06; blade angle at three-quarter radius, $60.5^{\circ}$.

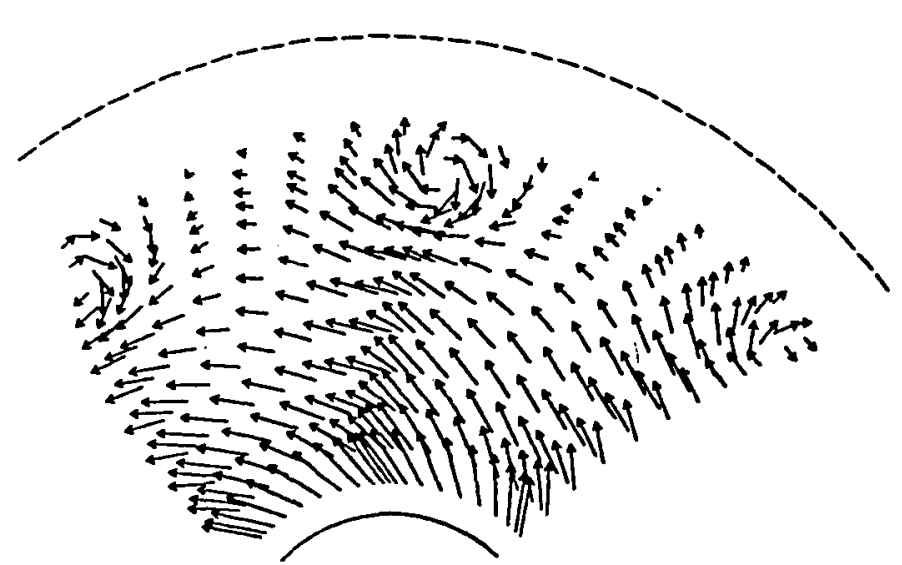

Figure 13. - Predicted secondary flow velocity vectors downstream of the SR-3 propeller. Free stream Mach number, 0.80 ; advance ratio, 3.06; blade angle at three-quarter radius, $61.3^{\circ}$. 


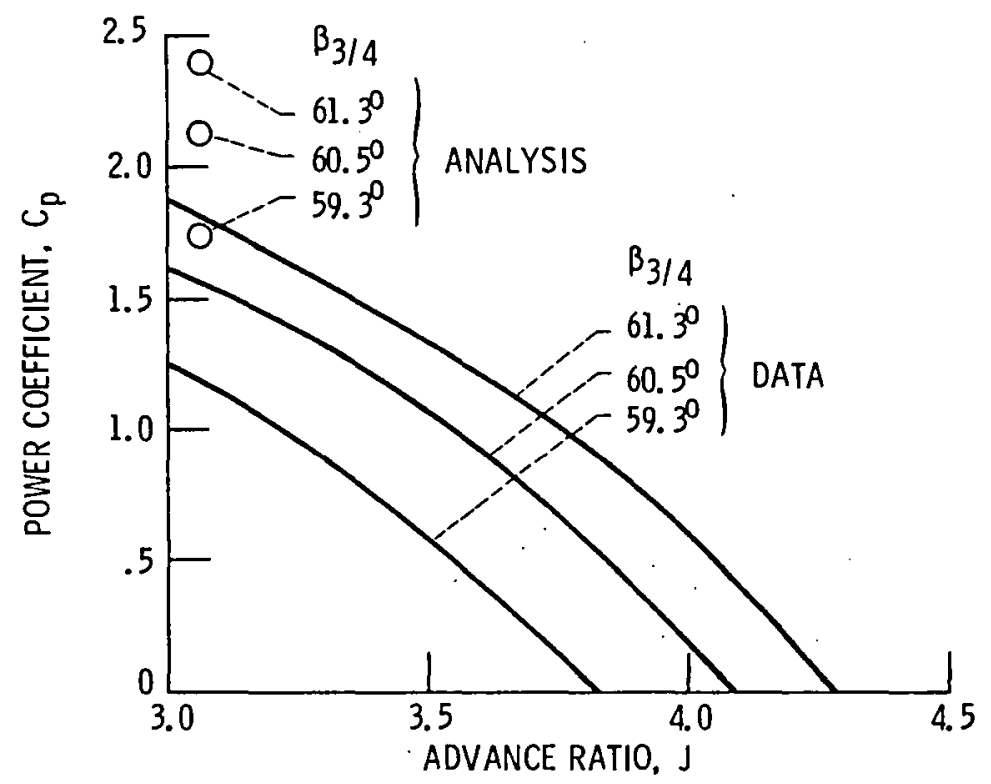

Figure 14. Comparison of predicted and measured power coefficient for the SR-3 propeller. Free stream Mach number, 0.8; advance ratio, 3.06. 


\begin{tabular}{|c|c|c|c|c|}
\hline $\begin{array}{l}\text { 1. Report No. NASA TM-83065 } \\
\text { AIAA-83-0188 }\end{array}$ & \multicolumn{2}{|c|}{ 2. Government Accession No. } & \multicolumn{2}{|c|}{ 3. Recipient's Catalog No." } \\
\hline \multicolumn{3}{|l|}{ 4. Title and Subtitle } & \multicolumn{2}{|l|}{ 5. Report Date } \\
\hline \multicolumn{3}{|c|}{$\begin{array}{l}\text { PREDICTION OF HIGH SPEED PROPELLER FLOW FIELDS } \\
\text { USING A THREE-DIMENSIONAL EULER ANAL YSIS }\end{array}$} & \multicolumn{2}{|c|}{$\begin{array}{l}\text { 6. Performing Organization Code } \\
535-03-12\end{array}$} \\
\hline \multicolumn{3}{|c|}{$\begin{array}{l}\text { 7. Author(s) } \\
\text { Lawrence J. Bober, Denny S. Chaussee, and Paul Kutler }\end{array}$} & \multicolumn{2}{|c|}{$\begin{array}{l}\text { 8. Performing Organization Report No. } \\
\text { E-1536 }\end{array}$} \\
\hline \multicolumn{3}{|c|}{ 9. Performing Organization Name and Address } & \multicolumn{2}{|l|}{ 10. Work Unit No. } \\
\hline \multicolumn{3}{|c|}{$\begin{array}{l}\text { National Aeronautics and Space Administration } \\
\text { Lewis Research Center }\end{array}$} & \multicolumn{2}{|c|}{ 11. Contract or Grant No. } \\
\hline Cleveland, Ohio 44135 & & & \multicolumn{2}{|c|}{$\begin{array}{l}\text { 13. Type of Report and Period Covered } \\
\text { Technical Memorandum }\end{array}$} \\
\hline \multicolumn{3}{|c|}{$\begin{array}{l}\text { 12. Sponsoring Agency Name and Address } \\
\text { National Aeronautics and Space Administration } \\
\text { Washington, D.C. } 20546\end{array}$} & \multicolumn{2}{|c|}{ 14. Sponsoring Agency Code } \\
\hline \multicolumn{5}{|c|}{$\begin{array}{l}\text { 15. Supplementary Notes Lawrence J. Bober, NASA Lewis Research Center; Denny S. Chaussee and } \\
\text { Paul Kutler, NASA Ames Research Center, Moffett Field, California. Prepared for the } \\
\text { Twenty-first Aerospace Sciences Conference sponsored by the American Institute of Aeronautics } \\
\text { and Astronautics, Reno, Nevada, January } 10-13,1983 \text {. }\end{array}$} \\
\hline \multicolumn{5}{|c|}{$\begin{array}{l}\text { 16. Abstract } \\
\text { To overcome the limitations of classical propeller theory, a computer program, NASPROP-E, } \\
\text { has been developed which solves for the flow field surrounding a multi-bladed propeller and } \\
\text { axisymmetric nacelle combination using a finite-difference method. The governing equations } \\
\text { are the three-dimensional unsteady Euler equations written in a cylindrical coordinate system. } \\
\text { They are marched in time until a steady state solution is obtained. The Euler equations require } \\
\text { no special treatment to model the blade work vorticity. The equations are solved using an } \\
\text { implicit approximate factorization method. Numerical results are presented which have } \\
\text { greatly increased the understanding of high speed propeller flow fields. Numerical results for } \\
\text { swirl angle downstream of the propeller and propeller power coefficient are higher than } \\
\text { experimental results. The radial variation of coefficient are higher than experimental results. } \\
\text { The radial variation of swirl angle, however, is in reasonable agreement with the experimental } \\
\text { results. The predicted variation of power coefficient with blade angle agrees very well with } \\
\text { data. }\end{array}$} \\
\hline $\begin{array}{l}\text { 17. Key Words (Suggested by Author(s) } \\
\text { Propellers; High speed } \\
\text { Euler equations; Numer }\end{array}$ & & $\begin{array}{l}\text { 18. Distribution S } \\
\text { Unclassif } \\
\text { STAR Cat }\end{array}$ & unlimited & \\
\hline $\begin{array}{l}\text { 19. Security Classif. (of this report) } \\
\text { Unclassified }\end{array}$ & 20. Ser & $\begin{array}{l}\text { of this pagel } \\
\text { sified }\end{array}$ & 21. No. of Pages & 22. Price" \\
\hline
\end{tabular}

' For sale by the National Technical Information Service, Springfield. Virgınia 22161 
End of Document 